\title{
Functional Promiscuity of Homologues of the Bacterial ArsA ATPases
}

\author{
Rostislav Castillo and Milton H. Saier Jr. \\ Division of Biological Sciences, University of California at San Diego, La Jolla, CA 92093-0116, USA \\ Correspondence should be addressed to Milton H. Saier Jr., msaier@ucsd.edu
}

Received 3 July 2010; Accepted 7 September 2010

Academic Editor: Ingolf Figved Nes

Copyright ( $) 2010$ R. Castillo and M. H. Saier Jr. This is an open access article distributed under the Creative Commons Attribution License, which permits unrestricted use, distribution, and reproduction in any medium, provided the original work is properly cited.

The ArsA ATPase of E. coli plays an essential role in arsenic detoxification. Published evidence implicates ArsA in the energization of As(III) efflux via the formation of an oxyanion-translocating complex with ArsB. In addition, eukaryotic ArsA homologues have several recognized functions unrelated to arsenic resistance. By aligning ArsA homologues, constructing phylogenetic trees, examining ArsA encoding operons, and estimating the probable coevolution of these homologues with putative transporters and auxiliary proteins unrelated to ArsB, we provide evidence for new functions for ArsA homologues. They may play roles in carbon starvation, gas vesicle biogenesis, and arsenic resistance. The results lead to the proposal that ArsA homologues energize four distinct and nonhomologous transporters, ArsB, ArsP, CstA, and Acr3.

\section{Introduction}

Arsenical species present threats to all organisms. The two predominant states of inorganic arsenic are arsenate $[\mathrm{As}(\mathrm{V})]$ and arsenite $[\mathrm{As}(\mathrm{III})]$. As(V) disrupts the cellular energy machinery as a phosphate analog, uncoupling energy production. The even more toxic As(III) binds the sulfhydryl groups of cysteine residues and inactivates proteins [1]. A variety of defenses have evolved against arsenic toxicity. Plasmid- and chromosome-borne ars operons encode genes including $\operatorname{ars} A,-B,-C,-D,-H,-I,-P$, and $-R$.

ars $C$ encodes an arsenate reductase that transfers electrons to As(V), reducing it to As(III), the efflux pump substrate [2]. ArsD is a metallochaperone which sequesters arsenite and antimonite and transfers them to the ArsA ATPase increasing the apparent affinity of ArsA for its substrates and lowering the concentration of free As(III) and $\mathrm{Sb}(\mathrm{III})$ in the cytosol [3]. ArsH, which is related to NADPH-dependent FMN reductases, has been implicated in arsenic resistance, but its precise biochemical function is not yet known [4]. arsP encodes a putative transmembrane permease which may play an ill-defined role in arsenic resistance in some organisms [5].
ArsB is a $12 \alpha$-helix transmembrane spanning (TMS) pump extruding As(III) and Sb(III) [6]. Transport via ArsB can be energized by the pmf or by forming an oxyaniontranslocating complex with the catalytic ArsA subunit, coupling ATP hydrolysis to efflux [7]. The characterized E. coli ArsA, a 583-amino acid (aa) ATPase, is composed of two homologous domains, A1 and A2, joined by a flexible linker. A1 and A2 are undoubtedly the products of a tandem intragenic duplication event. As(III) and Sb(III) form covalent bonds at the metal binding sites in A1 and A2, bringing the two domains together [8]. ArsA is allosterically activated by arsenite and antimonite binding, causing the nucleotide binding domains (NBDs) from each homologous half to come together to bind and hydrolyze ATP. Loss of either domain causes ArsA to lose catalytic function [9]. Prokaryotic ArsA has been extensively studied; however recent investigations of eukaryotic ArsA homologs, ASNA-1 (TRC40) and Arr4p, have furnished a variety of new roles for this family of proteins [10-15].

Previous studies have revealed that ATPases that energize macromolecular secretion can be found associated with a variety of evolutionarily distinct export systems. For example, the family of ATPases that energize bacterial 
conjugation (the transfer of DNA from a male donor to a female recipient) can also be found in a variety of other secretion systems. In addition to energizing conjugation via a Type IV secretion system, or its assembly, these ATPases can function in protein toxin secretion, bacterial pilus biogenesis, Type II protein secretion, and archaeal flagellar biogenesis. These facts reveal the promiscuity of ATPase energizers in the ultimate functional roles which have evolved over time [16]. Another example of functional promiscuity can be found in the ABC superfamily, where a single receptor can function with multiple transporters and a single transporter can utilize multiple receptors, thereby broadening substrate specificity (see the ABC superfamily; TC no. 3.A.1.1-34 in the Transporter Classification Database, http://www.tcdb.org/). Thus, "evolution is a tinker that cobbles together new functions from old ones, and the genome is a kind of parts bin of recyclable elements" (P. Z. Meyers).

Recently, established studies and our own analyses prompted further scrutiny of ArsA for uncharacterized roles in prokaryotes. We have conducted bioinformatic analyses using SEED, which revealed that different arsA homologues are often associated with genes for gas vesicles biogenesis and carbon starvation. Carbon starvation gene A ( cst $A)$ is often associated with an "auxiliary" genes cst $X$, and sometimes in archaea with $c s t Y$. ars A homologues are commonly present in operons that lack ars $B$ but contain $a c r 3$, a gene encoding an evolutionarily distinct As(III) efflux pump [17]. We include phylogenetic analyses of ArsA, ArsB, Acr3, ArsP, CstA, ArsA, CstX, and CstY, demonstrating that some but not others apparently coevolved. We identify new ars operon determinants encoding CcdA, thioredoxin, and redox-active disulfide protein 2 homologues. Finally, we have investigated the extent to which ars $P$ homologues are encoded in prokaryotic operons together with ars $A$ homologues. Our results suggest that ArsA plays roles in addition to those previously recognized.

\section{Materials and Methods}

2.1. Phylogenetic Tree Construction. A TCDB [18-20] ArsA query sequence was used in a default cutoff PSI-BLAST [21] search of the NCBI protein database to identify homologues. These were then screened using MakeTable5 [22] with a $90 \%$ cutoff to remove redundancies, close sequences, and fragmentary sequences. The CLUSTAL X program [23] was used to create multiple alignments of the homologue sequences retained by MakeTable5, and TREEVIEW [24] was used to draw phylogenetic trees generated from CLUSTALX alignments. 16S and 18S rRNA sequences were obtained from NCBI.

2.2. Superfamily Analyses. The Superfamily Tree program SFT1 [22] provided the evolutionary relationships of ArsA to other homologues using the neighbor-joining method. Representative proteins for each family were selected following an NCBI BLASTP search for sequences with 30\%-50\% identity and $e$-values less than $e^{-60}$. These same sequences were analyzed using ProtPars, which uses the parsimony method to predict protein relationships [25].

2.3. TMS Predictions. To predict TMSs, the WHAT program [26] was used to plot hydropathy, and the AveHAS program [27] was used to derive average hydropathy, amphipathicity, and similarity plots. These updated programs were used with the default sliding window of 19 residues.

2.4. SEED Analyses. Genome context analyses were performed with the SEED comparative genomics database $[28,29]$.This database can be found at http://theseed.uchicago .edu/ FIG $/$ SubsysEditor.cgi ? page $=$ ShowSpreadsheet $\&$ subsystem=CstA_Experiment and http://theseed.uchicago.edu/ FIG/SubsysEditor.cgi page $=$ ShowSpreadsheet $\&$ subsystem $=$ Arsenic_and_Antimonite_Resistance. Proteins were excluded from these analyses if they were not represented in the SEED database, or if they occurred in ars operons with low frequency. These included redox proteins of unknown function and potential modifying enzymes such as acetyltransferases. Operons were delineated based on SEED predictions. The putative functions of proteins were predicted by operon context analyses which included functional assignments based on coregulated genes. To study coevolution between proteins, we used SEED to select pairs of proteins that were encoded within the same operon.

2.5. MEME Analyses. The MEME program [30], with default settings, was used to search for ungapped conserved residue motifs with the ArsA homologues that we identified using PSI-BLAST as our training set.

\section{Results}

3.1. The Phylogeny of ArsA and arsA Gene Associations. Using E. coli ArsA as the query sequence, a PSI-BLAST search of the NCBI protein database brought up homologues which were screened using MakeTable5 [22] with a 90\% cutoff to remove redundancies, close sequences, and fragmentary sequences. These homologues are listed in Table 1. The CLUSTAL $\mathrm{X}$ program [23] was used to create a multiple alignment, and TREEVIEW [24] was used to draw the phylogenetic tree (Figure 1). The tree is divided into 18 clusters. These proteins can be found in Table 1, first, according to the cluster number, and second, according to their positions within the cluster.

To identify the potential functions of the proteins of each cluster, we used SEED to perform genome context analyses for organisms in the SEED genome database. Based on the pattern of association of the genes in each of the clusters, we have identified connections between various ars $A$ homologues and other previously unrelated genes.

Cluster 1 is composed of 55 bacterial and archaeal ArsA homologues, including the well-characterized E. coli ArsA protein, with an average size of 589 aas. Of the 24 cluster 1 proteins in SEED, 19 are encoded within operons containing homologues of $\operatorname{ars} B, \operatorname{acr} 3$, and/or ars $P$. Of the remaining five, four are next to an $a r s D$ homologue, three are located in operons with ars $R$ homologues, and three have $\operatorname{ars} B, a c r 3$, or 
$\operatorname{ars} P$ homologues elsewhere in the genome. The high degree of association of arsA homologues in cluster 1 with other ars operon determinants and the similarities in sizes between these proteins and the E. coli ArsA suggest that most Cluster 1 proteins function as energizers equivalent to the $E$. coli ArsA protein.

Cluster 2 is composed of seven archaeal ArsA homologues. SEED analysis was performed on the six members of this cluster included in the SEED database. Of these, none is found in the context of any of the typical ars operon genes, but four have acr 3 or ars $P$ homologues elsewhere in the genome. The tightly clustering proteins, Mja Mma2 and Mae2, are the three which have arsP genes elsewhere in the genome, while Msm1, clustering separately from the other three proteins, has an acr 3 and an ars $P$ elsewhere in the genome. The remaining proteins are encoded in genomes that lack any other ars transporter genes. Cluster 2 ArsA proteins are composed of a single ArsA domain with an average size of 337 aas. Thus, if they function catalytically like the E. coli ArsA protein, they must dimerize. With no consistent pattern of associated genes, the functions of cluster 2 ArsA homologues remain unknown.

Thirty-nine eukaryotic ArsA homologues comprising Cluster 3 have an average size of 345 aas. SEED analysis is only applicable for the S. cerevisiae genome; however, inspection of the region surrounding the arsA homologue did not identify functionally relevant proteins. Published evidence identifies these proteins as Arr4p and ASNA-1 homologues. While Arr4p from Saccharomyces cerevisiae is a member of the GET complex which is involved in trafficking from the Golgi apparatus to the ER and posttranslational insertion into the ER [14], homologues have been implicated in $\mathrm{As}(\mathrm{III})$ and $\mathrm{Sb}(\mathrm{III})$ resistance [15], metal and heat tolerance [13], regulation of Geflp to prevent copper accumulation [12], and regulation of insulin secretion [10]. Based on these results, we presume that these single domain ArsA homologues serve a diversity of biological functions, either energizing or regulating various transport processes.

Cluster 4 consists of eight bacterial ArsA homologues. Four of these are present in SEED. Each of these four genes is in an operon together with gvpFGJKLMNOW, encoding proteins involved in gas vesicle biogenesis, as well as a heat shock protein Hsp20 [31]. None of these four genes is present in an operon with other ars gene homologues, although acr3 and ars $P$ homologues are located elsewhere in three of these genomes. The average size of Cluster 4 proteins is 637 aas, similar to that of the E. coli ArsA. These observations suggest that these two domain homologues may function to energize some aspect of gas vesicle biogenesis.

Cluster 5 contains ten eukaryotic ArsA homologues with an average size of 401 aas. The functions of these proteins may be similar to the functions of those in Cluster 3, but no literature exists elucidating the roles of these ArsA homologues.

The two bacterial ArsA homologues of Cluster 6 have an average size of 645 aas. A SEED analysis revealed that the gene encoding Abal but not Mxal is associated with gas vesicle genes.
Cluster 7 contains six archaeal ArsA homologues with an average size of 342 aas. Three proteins from this cluster are in the SEED database. Nph1 is encoded by a gene present in an operon with the carbon starvation induced genes, $c s t A$ and $c s t X$. The other two homologues are not associated with genes that provide clues as to function.

Clusters 8-13 all consist of single domain proteins with average sizes of $396,389,410,424,361$, and 292, respectively. Representatives of four of these six clusters were present in SEED, and in every such case, the genomic content was analyzed. In no case did these analyses provide clues as to function. In a few cases, acr 3 and $\operatorname{ars} P$ genes were found elsewhere in the genomes, but not in close proximity to the genes encoding ArsA homologues. Thus, in these clusters, we could not provide functional predictions.

Cluster 14 contains six archaeal ArsA homologues with an average size of 327 aas. Three of these are in SEED. All three are in operons encoding CstA homologues, a single TMS ( $\sim 90$ aas) CstX and a single TMS ( $\sim 210$ aas) CstY. None is found in operons with ars operon determinants. Based on observations from Clusters 7 and 14-18, ArsA homologues that are associated with a CstA and a CstX tend to have single ATPase domains and a size of $\sim 330$ aas. The evidence suggests that proteins from this cluster function with CstA, CstX, and CstY. While CstA is a putative transporter, CstX and CstY may be auxiliary subunits [32-34]. Possibly the ArsA homologue energizes transport via CstA, thereby influencing the carbon starvation response.

The four bacterial and archaeal ArsA homologues of Cluster 15 have an average size of 336 aas. All four are encoded in operons with CstA and CstX. These two pairs of ArsA homologues are adjacent genes in two organisms with a sequence identity of 31\% for Par and 25\% sequence identity for Sth. The context and size of the proteins in this cluster indicate that they function with CstA and CstX (but not $\mathrm{Cst} \mathrm{Y}$ ) homologues.

Of the three bacterial ArsA homologues in Cluster 16, two are in SEED. One is encoded within an operon containing CstA and CstX, but the other is not found in the context of functionally significant genes. The average size of the three ArsA homologues, 306 aas, and the context of one ArsA encoding gene suggest that this cluster is composed of proteins functioning with CstA and CstX in the carbon starvation response.

There are 12 bacterial and archaeal ars $A$ homologues in Cluster 17 with an average protein size of 326 aas. Nine are found in SEED. All are located in gene clusters also encoding CstA and CstX. Interestingly, one ArsA-like protein is 426 aas long. The extra residues, at the $\mathrm{N}$-terminus, showed no sequence similarity with anything in the NCBI database. The context and average size of the genes encoding these proteins indicate that this cluster, like Cluster 16, functions with CstA and CstX.

Cluster 18 is composed of seven bacterial ArsA homologues with an average size of 336 aas. Of the four found in SEED, all are encoded by genes in operons that also encode CstA and CstX.

Thus, Clusters 14-18 and possibly 7 (based on one example) may all be concerned with carbon starvation 
TABle 1: ArsA homologues used to generate the phylogenetic tree. From left to right, the proteins are characterized according to cluster and abbreviation, organismal source, protein size, organismal type, and gi number. Within each cluster, proteins are presented according to position going clockwise around the tree.

\begin{tabular}{|c|c|c|c|c|}
\hline $\begin{array}{l}\text { Cluster and protein } \\
\text { abbreviations }\end{array}$ & Organismal source & $\begin{array}{l}\text { Protein size (no. } \\
\text { of aas) }\end{array}$ & Organismal type & GI no. \\
\hline \multicolumn{5}{|l|}{ Cluster 1} \\
\hline Sep1 & Staphylococcus epidermidis RP62A & 565 & Firmicutes & 57865830 \\
\hline Esp2 & Exiguobacterium sp. AT1b & 585 & Firmicutes & 187604364 \\
\hline Lsp2 & Lysinibacillus sphaericus C3-41 & 586 & Firmicutes & 169828142 \\
\hline Lpl1 & Lactobacillus plantarum WCFS1 & 576 & Firmicutes & 54307196 \\
\hline Sdy1 & Streptococcus dysgalactiae subsp. equisimilis & 580 & Firmicutes & 157419736 \\
\hline Lla1 & Lactococcus lactis subsp. cremoris MG1363 & 571 & Firmicutes & 125624073 \\
\hline Hin 1 & Haemophilus influenzae PittHH & 597 & $\gamma$ & 145636206 \\
\hline Ahyl & Anaerococcus hydrogenalis DSM 7454 & 580 & Firmicutes & 212696774 \\
\hline Bth1 & Bacteroides thetaiotaomicron VPI-5482 & 570 & Bacteroidetes & 29345526 \\
\hline Bcol & Bacillus coagulans 36D1 & 590 & Firmicutes & 124520553 \\
\hline Swo1 & $\begin{array}{l}\text { Syntrophomonas wolfei subsp. wolfei str. } \\
\text { Goettingen }\end{array}$ & 583 & Firmicutes & 114566470 \\
\hline Cte6 & Clostridium tetani E88 & 589 & Firmicutes & 28211528 \\
\hline Cph2 & Clostridium phytofermentans ISDg & 582 & Firmicutes & 160880289 \\
\hline Dha1 & Desulfitobacterium hafniense Y51 & 598 & Firmicutes & 89897344 \\
\hline Aor1 & Alkaliphilus oremlandii OhILAs & 582 & Firmicutes & 158320145 \\
\hline Cbe1 & Clostridium beijerinckii NCIMB 8052 & 582 & Firmicutes & 150016979 \\
\hline Bse1 & Bacillus selenitireducens MLS10 & 587 & Firmicutes & 163763208 \\
\hline Bce2 & Bacillus cereus ATCC 10987 & 586 & Firmicutes & 44004495 \\
\hline Bsp2 & Bacillus sp. SG-1 & 594 & Firmicutes & 149180106 \\
\hline Gur1 & Geobacter uraniireducens Rf4 & 583 & $\delta$ & 148263449 \\
\hline Bph1 & Burkholderia phytofirmans PsJN & 584 & $\beta$ & 187925677 \\
\hline Mma1 & Magnetospirillum magnetotacticum MS-1 & 574 & $\alpha$ & 46201533 \\
\hline Rru1 & Rhodospirillum rubrum ATCC 11170 & 571 & $\alpha$ & 83592783 \\
\hline Bvil & Burkholderia vietnamiensis G4 & 587 & $\beta$ & 134293367 \\
\hline Asp4 & Azoarcus sp. BH72 & 582 & $\beta$ & 119898647 \\
\hline Rbal & Rhodopirellula baltica SH 1 & 593 & Planctomycetes & 32475953 \\
\hline Aeh2 & Alkalilimnicola ehrlichei MLHE-1 & 571 & $\gamma$ & 114321857 \\
\hline Orf3 & delta proteobacterium MLMS-1 & 592 & $\delta$ & 94266138 \\
\hline Vba1 & Verrucomicrobiae bacterium DG1235 & 584 & Verrucomicrobia & 198257295 \\
\hline Vha1 & Vibrio harveyi ATCC BAA-1116 & 582 & $\gamma$ & 156973785 \\
\hline Styl & Salmonella typhimurium & 585 & $\gamma$ & 32470159 \\
\hline Ahy2 & $\begin{array}{l}\text { Aeromonas hydrophila subsp. hydrophila } \\
\text { ATCC } 7966\end{array}$ & 599 & $\gamma$ & 117618702 \\
\hline Ppr1 & Photobacterium profundum SS9 & 590 & $\gamma$ & 54308735 \\
\hline Psp1 & Psychromonas sp. CNPT3 & 602 & $\gamma$ & 90408036 \\
\hline Cfe1 & Chlorobium ferrooxidans DSM 13031 & 591 & Chlorobi & 110598505 \\
\hline Psp2 & Pseudomonas sp. TS44 & 585 & $\gamma$ & 170026534 \\
\hline Rfe1 & Rhodoferax ferrireducens T118 & 589 & $\beta$ & 89902424 \\
\hline Dar1 & Dechloromonas aromatica RCB & 590 & $\beta$ & 71908250 \\
\hline Afal & Alcaligenes faecalis & 591 & $\beta$ & 42741719 \\
\hline Pae1 & Pseudomonas aeruginosa & 588 & $\gamma$ & 187939925 \\
\hline
\end{tabular}


TABle 1: Continued.

\begin{tabular}{|c|c|c|c|c|}
\hline $\begin{array}{l}\text { Cluster and protein } \\
\text { abbreviations }\end{array}$ & Organismal source & $\begin{array}{c}\text { Protein size (no. } \\
\text { of aas) }\end{array}$ & Organismal type & GI no. \\
\hline Sen 1 & $\begin{array}{l}\text { Salmonella enterica subsp. enterica serovar } \\
\text { Saintpaul str. SARA29 }\end{array}$ & 586 & $\gamma$ & 167553198 \\
\hline Aar1 & Aromatoleum aromaticum EbN1 & 592 & $\beta$ & 56478279 \\
\hline Acal & Acidithiobacillus caldus & 612 & $\gamma$ & 60686969 \\
\hline Otr1 & Ochrobactrum tritici & 582 & $\alpha$ & 94483089 \\
\hline Pst2 & Providencia stuartii ATCC 25827 & 594 & $\gamma$ & 188026271 \\
\hline Ssp2 & Shewanella sp. ANA-3 & 588 & $\gamma$ & 117920786 \\
\hline Yin1 & Yersinia intermedia ATCC 29909 & 586 & $\gamma$ & 77979455 \\
\hline Asp1 & Arthrobacter sp. FB24 & 621 & Actinobacteria & 116668777 \\
\hline Mgi1 & Mycobacterium gilvum PYR-GCK & 589 & Actinobacteria & 145222246 \\
\hline Rer1 & Rhodococcus erythropolis & 585 & Actinobacteria & 33867198 \\
\hline Rle1 & Rhizobium leguminosarum bv. viciae 3841 & 588 & $\alpha$ & 116254241 \\
\hline Pnal & Polaromonas naphthalenivorans CJ2 & 600 & $\beta$ & 121606619 \\
\hline Asp2 & Acidovorax sp. JS42 & 586 & $\beta$ & 121594853 \\
\hline Hla2 & Halorubrum lacusprofundi ATCC 49239 & 640 & Euryarchaeota & 153896608 \\
\hline Hsp4 & Halobacterium sp. NRC-1 & 644 & Euryarchaeota & 10803670 \\
\hline \multicolumn{5}{|l|}{ Cluster 2} \\
\hline Mka1 & Methanopyrus kandleri AV19 & 333 & Euryarchaeota & 20095116 \\
\hline Mst1 & Methanosphaera stadtmanae DSM 3091 & 328 & Euryarchaeota & 84488998 \\
\hline Msm1 & Methanobrevibacter smithii ATCC 35061 & 340 & Euryarchaeota & 148643230 \\
\hline Mth1 & $\begin{array}{l}\text { Methanothermobacter thermautotrophicus str. } \\
\text { Delta H }\end{array}$ & 324 & Euryarchaeota & 15679508 \\
\hline Mjal & Methanocaldococcus jannaschii DSM 2661 & 349 & Euryarchaeota & 15669329 \\
\hline Mma2 & Methanococcus maripaludis S2 & 345 & Euryarchaeota & 45357726 \\
\hline Mae2 & Methanococcus aeolicus Nankai-3 & 341 & Euryarchaeota & 150401428 \\
\hline \multicolumn{5}{|l|}{ Cluster 3} \\
\hline Tval & Trichomonas vaginalis G3 & 297 & Trichomonada & 123416597 \\
\hline Tva2 & Trichomonas vaginalis G3 & 292 & Trichomonada & 123451254 \\
\hline Yli1 & Yarrowia lipolytica CLIB122 & 327 & Fungi & 50554649 \\
\hline Gze1 & Gibberella zeae PH-1 & 341 & Fungi & 46136751 \\
\hline Ani1 & Aspergillus nidulans FGSC A4 & 340 & Fungi & 67524903 \\
\hline Spo1 & Schizosaccharomyces pombe & 329 & Fungi & 19115182 \\
\hline Rgl1 & Rhodotorula glutinis & 339 & Fungi & 183396512 \\
\hline Uma1 & Ustilago maydis 521 & 332 & Fungi & 71019509 \\
\hline Ccil & Coprinopsis cinerea okayama7\#130 & 326 & Fungi & 169843560 \\
\hline Cne1 & $\begin{array}{l}\text { Cryptococcus neoformans var. neoformans } \\
\text { JEC21 }\end{array}$ & 325 & Fungi & 58260906 \\
\hline Cal1 & Candida albicans SC5314 & 350 & Fungi & 68468811 \\
\hline Scel & Saccharomyces cerevisiae & 354 & Fungi & 51013779 \\
\hline Tad1 & Trichoplax adhaerens & 339 & Metazoa & 196008131 \\
\hline Ppa2 & Physcomitrella patens subsp. patens & 365 & Viridiplantae & 168012492 \\
\hline Obr1 & Oryza brachyantha & 364 & Viridiplantae & 110430665 \\
\hline Ath2 & Arabidopsis thaliana & 345 & Viridiplantae & 8570442 \\
\hline Psil & Picea sitchensis & 374 & Viridiplantae & 116784166 \\
\hline Ddil & Dictyostelium discoideum AX4 & 329 & Amoebozoa & 66800287 \\
\hline Apil & Acyrthosiphon pisum & 339 & Metazoa & 193582608 \\
\hline Dre1 & Danio rerio & 341 & Metazoa & 50539666 \\
\hline
\end{tabular}


TABle 1: Continued.

\begin{tabular}{|c|c|c|c|c|}
\hline $\begin{array}{l}\text { Cluster and protein } \\
\text { abbreviations }\end{array}$ & Organismal source & $\begin{array}{l}\text { Protein size (no. } \\
\text { of aas) }\end{array}$ & Organismal type & GI no. \\
\hline Nve1 & Nematostella vectensis & 334 & Metazoa & 156398556 \\
\hline Spul & Strongylocentrotus purpuratus & 346 & Metazoa & 72050675 \\
\hline Dan1 & Drosophila ananassae & 336 & Metazoa & 194755601 \\
\hline Nvil & Nasonia vitripennis & 344 & Metazoa & 156537421 \\
\hline Aae3 & Aedes aegypti & 341 & Metazoa & 157128460 \\
\hline Tcal & Tribolium castaneum & 330 & Metazoa & 91081505 \\
\hline Cel1 & Caenorhabditis elegans & 342 & Metazoa & 17557003 \\
\hline Bmal & Brugia malayi & 344 & Metazoa & 170590260 \\
\hline Pte1 & Paramecium tetraurelia strain $\mathrm{d} 4-2$ & 325 & Oligohymenophorea & 145545770 \\
\hline Tth1 & Tetrahymena thermophila SB210 & 349 & Oligohymenophorea & 118401519 \\
\hline Tcr1 & Trypanosoma cruzi strain CL Brener & 359 & Trypanosomatidae & 71401129 \\
\hline Lmal & Leishmania major strain Friedlin & 409 & Trypanosomatidae & 157865666 \\
\hline Tpal & Theileria parva strain Muguga & 361 & Apicomplexa & 71027033 \\
\hline Bbol & Babesia bovis T2Bo & 358 & Apicomplexa & 156082722 \\
\hline Pbe1 & Plasmodium berghei str. ANKA & 379 & Apicomplexa & 68071753 \\
\hline Cmul & Cryptosporidium muris RN66 & 390 & Apicomplexa & 209879305 \\
\hline Cpal & Cryptosporidium parvum Iowa II & 366 & Apicomplexa & 126654216 \\
\hline Ehil & Entamoeba histolytica HM-1:IMSS & 327 & Amoebozoa & 67466277 \\
\hline Gla1 & Giardia lamblia ATCC 50803 & 354 & Hexamitidae & 159119999 \\
\hline \multicolumn{5}{|l|}{ Cluster 4} \\
\hline Gur2 & Geobacter uraniireducens Rf4 & 637 & $\delta$ & 148264869 \\
\hline Ssp1 & Synechococcus sp. JA-3-3Ab & 684 & Cyanobacteria & 86605793 \\
\hline Mae1 & Microcystis aeruginosa PCC 7806 & 633 & Cyanobacteria & 159025965 \\
\hline Nsp2 & Nostoc sp. PCC 7120 & 635 & Cyanobacteria & 17229736 \\
\hline Nsp3 & Nodularia spumigena CCY 9414 & 617 & Cyanobacteria & 119512417 \\
\hline Ama1 & Arthrospira maxima CS-328 & 637 & Cyanobacteria & 209527482 \\
\hline Ter2 & Trichodesmium erythraeum IMS101 & 626 & Cyanobacteria & 113475961 \\
\hline Npu1 & Nostoc punctiforme PCC 73102 & 623 & Cyanobacteria & 186682498 \\
\hline \multicolumn{5}{|l|}{ Cluster 5} \\
\hline Zma2 & Zea mays & 374 & Viridiplantae & 195625344 \\
\hline Ath3 & Arabidopsis thaliana & 391 & Viridiplantae & 30697424 \\
\hline Vvil & Vitis vinifera & 422 & Viridiplantae & 147852937 \\
\hline Ppal & Physcomitrella patens subsp. patens & 359 & Viridiplantae & 168024699 \\
\hline Zmal & Zea mays & 394 & Viridiplantae & 195645964 \\
\hline Ath1 & Arabidopsis thaliana & 411 & Viridiplantae & 30681260 \\
\hline Vvi2 & Vitis vinifera & 409 & Viridiplantae & 157343988 \\
\hline Ptr1 & Populus trichocarpa & 407 & Viridiplantae & 118487322 \\
\hline Cre1 & Chlamydomonas reinhardtii & 513 & Viridiplantae & 159488560 \\
\hline Olu1 & Ostreococcus lucimarinus CCE9901 & 330 & Viridiplantae & 145350244 \\
\hline \multicolumn{5}{|l|}{ Cluster 6} \\
\hline Aba1 & Acidobacteria bacterium Ellin345 & 634 & Acidobacteria & 94969437 \\
\hline Mxa1 & Myxococcus xanthus DK 1622 & 655 & $\delta$ & 108758691 \\
\hline \multicolumn{5}{|l|}{ Cluster 7} \\
\hline Hwal & Haloquadratum walsbyi DSM 16790 & 312 & Euryarchaeota & 110668350 \\
\hline Orf2 & uncultured prokaryote 2E01B & 314 & none & 85372676 \\
\hline Nph1 & Natronomonas pharaonis DSM 2160 & 317 & Euryarchaeota & 76801342 \\
\hline
\end{tabular}


Table 1: Continued.

\begin{tabular}{|c|c|c|c|c|}
\hline $\begin{array}{l}\text { Cluster and protein } \\
\text { abbreviations }\end{array}$ & Organismal source & $\begin{array}{l}\text { Protein size (no. } \\
\text { of aas) }\end{array}$ & Organismal type & GI no. \\
\hline Nph2 & Natronomonas pharaonis DSM 2160 & 370 & Euryarchaeota & 76801234 \\
\hline Hsp2 & Halobacterium sp. NRC-1 & 347 & Euryarchaeota & 15789625 \\
\hline Hla3 & Halorubrum lacusprofundi ATCC 49239 & 392 & Euryarchaeota & 153895127 \\
\hline \multicolumn{5}{|l|}{ Cluster 8} \\
\hline Cth5 & Chloroherpeton thalassium ATCC 35110 & 405 & Chlorobi & 193215201 \\
\hline Cau4 & Chloroflexus aurantiacus J-10-fl & 399 & Chloroflexi & 163845724 \\
\hline Ctel & Chlorobium tepidum TLS & 395 & Chlorobi & 21673808 \\
\hline Cth2 & Chloroherpeton thalassium ATCC 35110 & 405 & Chlorobi & 193214297 \\
\hline Pae2 & Prosthecochloris aestuarii DSM 271 & 404 & Chlorobi & 194332962 \\
\hline Cte3 & Chlorobium tepidum TLS & 398 & Chlorobi & 21672957 \\
\hline Plu2 & Pelodictyon luteolum DSM 273 & 406 & Chlorobi & 78185960 \\
\hline Cte2 & Chlorobium tepidum TLS & 405 & Chlorobi & 21674757 \\
\hline Cth4 & Chloroherpeton thalassium ATCC 35110 & 402 & Chlorobi & 193214011 \\
\hline Caul & Chloroflexus aurantiacus J-10-fl & 390 & Chloroflexi & 163846065 \\
\hline Daul & Candidatus Desulforudis audaxviator MP104C & 397 & Firmicutes & 169830523 \\
\hline Ame1 & Alkaliphilus metalliredigens QYMF & 391 & Firmicutes & 150389286 \\
\hline Bcel & Bacillus cereus ATCC 10987 & 392 & Firmicutes & 42779427 \\
\hline Rxyl & Rubrobacter xylanophilus DSM 9941 & 394 & Actinobacteria & 108804153 \\
\hline Pae3 & Prosthecochloris aestuarii DSM 271 & 395 & Chlorobi & 194333066 \\
\hline Cte4 & Chlorobium tepidum TLS & 394 & Chlorobi & 21674880 \\
\hline Cli1 & Chlorobium limicola DSM 245 & 395 & Chlorobi & 189347837 \\
\hline Sel1 & Synechococcus elongatus PCC 7942 & 392 & Cyanobacteria & 81300068 \\
\hline Gvil & Gloeobacter violaceus PCC 7421 & 394 & Cyanobacteria & 37520959 \\
\hline Adil & Angiococcus disciformis & 405 & $\delta$ & 53747901 \\
\hline Hsp1 & Hydrogenobaculum sp. Y04AAS1 & 397 & Aquificae & 195953882 \\
\hline Aae2 & Aquifex aeolicus VF5 & 396 & Aquificae & 15606091 \\
\hline Hsp5 & Hydrogenivirga sp. 128-5-R1-1 & 393 & Aquificae & 163783049 \\
\hline Sus1 & Solibacter usitatus Ellin6076 & 395 & Acidobacteria & 116624985 \\
\hline Dal1 & Desulfatibacillum alkenivorans AK-01 & 397 & $\delta$ & 163725491 \\
\hline Haul & Herpetosiphon aurantiacus ATCC 23779 & 391 & Chloroflexi & 159900394 \\
\hline Rsp1 & Roseiflexus sp. RS-1 & 396 & Chloroflexi & 148655082 \\
\hline Cau3 & Chloroflexus aurantiacus J-10-fl & 401 & Chloroflexi & 163848482 \\
\hline Cth3 & Chloroherpeton thalassium ATCC 35110 & 383 & Chlorobi & 193214006 \\
\hline Cte7 & Chlorobium tepidum TLS & 384 & Chlorobi & 21674751 \\
\hline \multicolumn{5}{|l|}{ Cluster 9} \\
\hline Mma4 & Microscilla marina ATCC 23134 & 390 & Bacteroidetes & 124004922 \\
\hline Pto1 & Picrophilus torridus DSM 9790 & 386 & Euryarchaeota & 48478270 \\
\hline Tac1 & Thermoplasma acidophilum DSM 1728 & 387 & Euryarchaeota & 16081559 \\
\hline Cph3 & Clostridium phytofermentans ISDg & 385 & Firmicutes & 160879841 \\
\hline Fnu2 & $\begin{array}{l}\text { Fusobacterium nucleatum subsp. nucleatum } \\
\text { ATCC } 25586\end{array}$ & 388 & Fusobacteria & 19704869 \\
\hline Cph1 & Clostridium phytofermentans ISDg & 393 & Firmicutes & 160879840 \\
\hline Fnul & $\begin{array}{l}\text { Fusobacterium nucleatum subsp. nucleatum } \\
\text { ATCC } 25586\end{array}$ & 396 & Fusobacteria & 19704870 \\
\hline
\end{tabular}


Table 1: Continued.

\begin{tabular}{|c|c|c|c|c|}
\hline $\begin{array}{l}\text { Cluster and protein } \\
\text { abbreviations }\end{array}$ & Organismal source & $\begin{array}{l}\text { Protein size (no. } \\
\text { of aas) }\end{array}$ & Organismal type & GI no. \\
\hline \multicolumn{5}{|l|}{ Cluster 10} \\
\hline Nsp1 & Nocardioides sp. JS614 & 410 & Actinobacteria & 119717335 \\
\hline Jsp1 & Janibacter sp. HTCC2649 & 421 & Actinobacteria & 84496160 \\
\hline Ser1 & Saccharopolyspora erythraea NRRL 2338 & 400 & Actinobacteria & 134098280 \\
\hline Nfa1 & Nocardia farcinica IFM 10152 & 436 & Actinobacteria & 54023683 \\
\hline Mab1 & Mycobacterium abscessus & 423 & Actinobacteria & 169629069 \\
\hline Mle1 & Mycobacterium leprae TN & 415 & Actinobacteria & 15827412 \\
\hline Msp1 & Mycobacterium sp. MCS & 421 & Actinobacteria & 108800245 \\
\hline Msm2 & Mycobacterium smegmatis str. MC2 155 & 425 & Actinobacteria & 118471162 \\
\hline \multicolumn{5}{|l|}{ Cluster 11} \\
\hline Cth1 & Chloroherpeton thalassium ATCC 35110 & 434 & Chlorobi & 193214353 \\
\hline Cte5 & Chlorobium tepidum TLS & 436 & Chlorobi & 21673187 \\
\hline Cau2 & Chloroflexus aurantiacus J-10-fl & 407 & Chloroflexi & 163845728 \\
\hline Cth6 & $\begin{array}{l}\text { Candidatus Chloracidobacterium } \\
\text { thermophilum }\end{array}$ & 420 & Acidobacteria & 157273534 \\
\hline \multicolumn{5}{|l|}{ Cluster 12} \\
\hline Ter1 & Trichodesmium erythraeum IMS101 & 364 & Cyanobacteria & 113474690 \\
\hline Bbal & Bdellovibrio bacteriovorus HD100 & 357 & $\delta$ & 42525090 \\
\hline \multicolumn{5}{|l|}{ Cluster 13} \\
\hline Mmy1 & $\begin{array}{l}\text { Mycoplasma mycoides subsp. mycoides LC str. } \\
\text { GM12 }\end{array}$ & 304 & Tenericutes & 188159217 \\
\hline Esp1 & Exiguobacterium sp. AT1b & 279 & Firmicutes & 187605346 \\
\hline \multicolumn{5}{|l|}{ Cluster 14} \\
\hline Ihol & Ignicoccus hospitalis KIN4/I & 309 & Crenarchaeota & 156938113 \\
\hline Sma1 & Staphylothermus marinus F1 & 329 & Crenarchaeota & 126465059 \\
\hline Tbal & Thermococcus barophilus MP & 330 & Euryarchaeota & 197627410 \\
\hline Tkol & Thermococcus kodakarensis KOD1 & 331 & Euryarchaeota & 57640929 \\
\hline Pab1 & Pyrococcus abyssi GE5 & 330 & Euryarchaeota & 14521447 \\
\hline Ton1 & Thermococcus onnurineus NA1 & 330 & Euryarchaeota & 212224056 \\
\hline \multicolumn{5}{|l|}{ Cluster 15} \\
\hline Par1 & Pyrobaculum arsenaticum DSM 13514 & 334 & Crenarchaeota & 145591126 \\
\hline Sth2 & Symbiobacterium thermophilum IAM 14863 & 339 & Firmicutes & 51891819 \\
\hline Par2 & Pyrobaculum arsenaticum DSM 13514 & 327 & Crenarchaeota & 145591125 \\
\hline Sth1 & Symbiobacterium thermophilum IAM 14863 & 345 & Firmicutes & 51891818 \\
\hline \multicolumn{5}{|l|}{ Cluster 16} \\
\hline Wsul & Wolinella succinogenes DSM 1740 & 313 & $\varepsilon$ & 34557874 \\
\hline Aae1 & Aquifex aeolicus VF5 & 299 & Aquificae & 15605857 \\
\hline Hsp3 & Hydrogenivirga sp. 128-5-R1-1 & 306 & Aquificae & 163782204 \\
\hline \multicolumn{5}{|l|}{ Cluster 17} \\
\hline Csa1 & Chromohalobacter salexigens DSM 3043 & 313 & $\gamma$ & 92114127 \\
\hline Bsp1 & Bacillus sp. NRRL B-14911 & 328 & Firmicutes & 89098562 \\
\hline Bhal & Bacillus halodurans C-125 & 313 & Firmicutes & 15614358 \\
\hline Oih1 & Oceanobacillus iheyensis HTE831 & 307 & Firmicutes & 23098830 \\
\hline Hhal & Halorhodospira halophila SL1 & 311 & $\gamma$ & 121998972 \\
\hline Aeh1 & Alkalilimnicola ehrlichei MLHE-1 & 318 & $\gamma$ & 114319474 \\
\hline
\end{tabular}


TABLe 1: Continued.

\begin{tabular}{|c|c|c|c|c|}
\hline $\begin{array}{l}\text { Cluster and protein } \\
\text { abbreviations }\end{array}$ & Organismal source & $\begin{array}{c}\text { Protein size (no. } \\
\text { of aas) }\end{array}$ & Organismal type & GI no. \\
\hline Tful & Thermobifida fusca YX & 301 & Actinobacteria & 72161795 \\
\hline Nmol & Nitrococcus mobilis Nb-231 & 311 & $\gamma$ & 88811608 \\
\hline Nph3 & Natronomonas pharaonis DSM 2160 & 318 & Euryarchaeota & 76801557 \\
\hline Hwa2 & Haloquadratum walsbyi DSM 16790 & 327 & Euryarchaeota & 110667012 \\
\hline Hla1 & Halorubrum lacusprofundi ATCC 49239 & 341 & Euryarchaeota & 153896540 \\
\hline Hma1 & Haloarcula marismortui ATCC 43049 & 426 & Euryarchaeota & 55379238 \\
\hline \multicolumn{5}{|l|}{ Cluster 18} \\
\hline Par3 & Psychrobacter arcticus 273-4 & 339 & $\gamma$ & 71065679 \\
\hline Psp3 & Psychrobacter sp. PRwf-1 & 331 & $\gamma$ & 148653012 \\
\hline Asp3 & Alcanivorax sp. DG881 & 347 & $\gamma$ & 196196123 \\
\hline Ilo1 & Idiomarina loihiensis L2TR & 336 & $\gamma$ & 56459808 \\
\hline Iba1 & Idiomarina baltica OS145 & 338 & $\gamma$ & 85712073 \\
\hline Pst1 & Pseudomonas stutzeri A1501 & 335 & $\gamma$ & 146280770 \\
\hline Bli1 & Brevibacterium linens BL2 & 327 & Actinobacteria & 62424272 \\
\hline
\end{tabular}

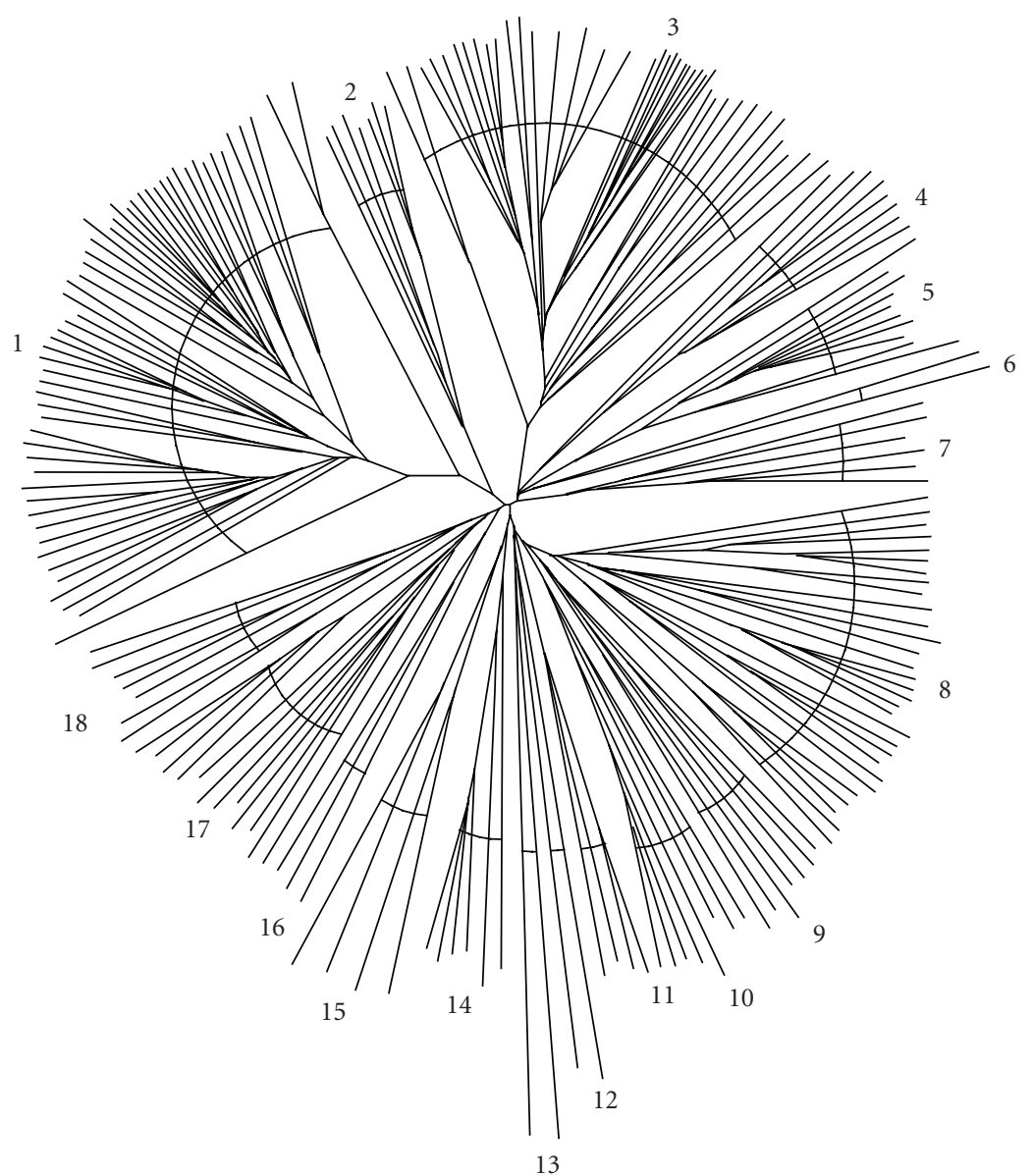

FIGURE 1: Phylogenetic tree of full length prokaryotic and eukaryotic ArsA homologues. The CLUSTAL X program [23] was used to create a multiple alignment of the protein sequences, while TreeView [24] generated the tree. The phylogenetic clusters are labeled 1-18 clockwise. Specific proteins can be found in Table 1, first according to cluster number and then according to position within the cluster. The dendrogram is shown in Figure S1. 
responses, functioning in conjunction with CstA, CstX, and CstY in cluster 14.

3.2. CstA Protein Associations. Using SEED, we have identified 26 instances in bacteria and archaea where CstA is associated with an ArsA homologue. In 24 of these instances CstX is found with CstA, but it is missing in one bacterium and one archaeon. CstX is never present without CstA. Five archaea analyzed also have CstY encoded within the cluster containg ArsA, CstA, and the CstX homologues. We suggest that CstA functions together with the ArsA and CstX homologues. Possibly CstX couples CstA-catalyzed transport to ArsA-dependent ATP hydrolysis as demonstrated for other transport systems [29]. It is important to note that these operons do not contain an arsR and thus are not likely to be regulated by the usual mechanism in response to the arsenite concentration.

Phylogenetic trees were generated for those CstA, CstX, CstY, and ArsA homologues that occur together within the same operons. Trees for the CstA and ArsA homologues are shown in Figures 2(a) and 2(b), respectively, for CstA and CstX homologues in Figures 2(c) and 2(d), respectively, and for ArsA and CstX homologues in Figures 2(e) and 2(f), respectively. Comparing Figures 2(a) and 2(b), we see that clustering patterns correspond within experimental error. Thus, Clusters 1 and 2 branch more closely from each other than they do to any other branch. These and all remaining clusters have the same protein compositions on the two trees. Most of these clusters branch from points near the centers of these trees, as is particularly apparent in Figure 2(b). It is interesting to note that the CstA tree shows tighter clustering than observed for the ArsA tree. Moreover, within the threeprotein cluster 3 and the five-protein cluster 4, branching patterns are the same in both trees. We therefore conclude that these two families of proteins have coevolved.

Comparing the phylogenetic tree for CstX (Figure 2(d)) with that for CstA (Figure 2(c)), we see that almost all proteins show excellent correspondence in the two trees. For example, at the bottom of both trees, we find the same clustering patterns for the two sets of proteins, except that each organism possesses a single CstA, but in two cases, there are two paralogous CstX proteins, Par11 and Par12, and Tko11 and Tko12. In both cases, it appears that the novel paralogue arose relatively early. Interestingly, Tko12 exhibits striking similarities with Pab1 and Sma1, while Tko11 has no such close ortholog (see Figure $2(\mathrm{~d})$ ). The top halves of the two trees similarly show clustering patterns largely consistent with coevolution. However, there are a couple of potential inconsistencies. Bli1 and Kse1 cluster together in Figure 2(c), but not in Figure 2(d). This observation is anomalous because in all other subclusters shown in these two trees, the proteins show shorter branch lengths in Figure 2(d) compared to those in Figure 2(c). Additionally, Wsul stems from the center of Figure $2(\mathrm{~d})$ tree but clusters more tightly with the top half of the tree in Figure 2(c). We therefore conclude that CstA and CstX evolved in parallel with just two potential exceptions. The results are consistent with coevolution and therefore with cofunctioning, possibly involving direct interaction.
Comparing the CstX phylogenetic trees (Figure 2(f)) with that for ArsA (Figure 2(e)), we see that almost all proteins in one tree correspond in position to those in the other tree. It appears that ArsA has diverged in sequence more rapidly than has $\mathrm{CstX}$, accounting for the greater branch lengths in the ArsA tree (Figure 2(e)). Potential exceptions are observed when Figures 2(e) and 2(f) are compared or when Figures 2(c) and 2(d) are compared. Thus, we observe the same anomaly regarding the inversion of Wsul with Bli1 as well as the clustering of Ksel with Bli1 in Figure 2(e) but not Figure 2(f). The correspondence between Figures 2(a) and 2(b) proved to be greater than that between Figures 2(c) and 2(d) or Figures 2(e) and 2(f). We suggest that CstX homologues might interact with both the ArsA and CstA homologues, possibly because CstX serves as the linker between the other two proteins.

An additional protein found within the operons of five archaea is the CstY protein. The CstY tree showed branching patterns almost identical to those of the CstA tree. Four of these five operons include the ars $A$ gene, and except for the absence of ArsA in Hyperthermus butylicus DSM 5456, we see that CstY also appeared to coevolve with CstA and ArsA. In like fashion, CstY seems to have coevolved with CstX except for the duplication in Thermococcus kodakarensis KOD1 (data not shown).

3.3. Gas Vesicle Biogenesis Proteins. Several ArsA homologues were encoded within clusters of genes concerned with gas vesicle biogenesis. These gene clusters were identified in four different phyla. Two organisms from the Bacteroides/Chlorobi phyla, Polaribacter irgensii 23-P and Pelodictyon luteolum DSM 273 possess gene clusters where the $\operatorname{ars} A$ homologue colocalized with the following genes: gvpNLFGJKM and $h s p 20$. Although the genes represented in the two clusters from the above organisms were the same, five and four copies of the paralogous $L$ and $F$ genes [35], and two and four copies of the $M$ gene were present in these two clusters, respectively. Most similar to these clusters was the cluster from the $\delta$-Proteobacterium, Geobacter uraniireducens Rf4. This cluster differs from the other two in that there are paralogues of the $L / F$ genes, two paralogues of the $M$ gene and an additional gene, the $O$ gene. The cyanobacterial cluster, represented by Anabaena variablis ATCC 29413 and Nostoc sp. PCC 7120, also resembles that found in the previously described organisms, except that $L / F$ genes are present in single copies, the $M$ and $h s p 20$ genes are lacking, and an additional gene, the $W$ gene, is present. Finally, in the acidobacterial phylum the ars $A$ homologue is accompanied by two copies of the $L / F$ genes and one copy each of the $J, K$, and $M$ genes.

Very little is known about the genes involved in gas vesicle biogenesis. However, all of the genes described above are soluble proteins except for GvpG and GvpM which each has a single TMS. GvpG, of about 126 aas, has an N-terminal TMS, while GvpM, of about 100 aas, has its TMS centrally located. GvpN is an ATPase of the AAA+ superfamily with both the Walker A and Walker B motifs. This protein has been reported to increase the production of gas vesicles [36]. GvpL/F have been reported to be nucleation proteins, 


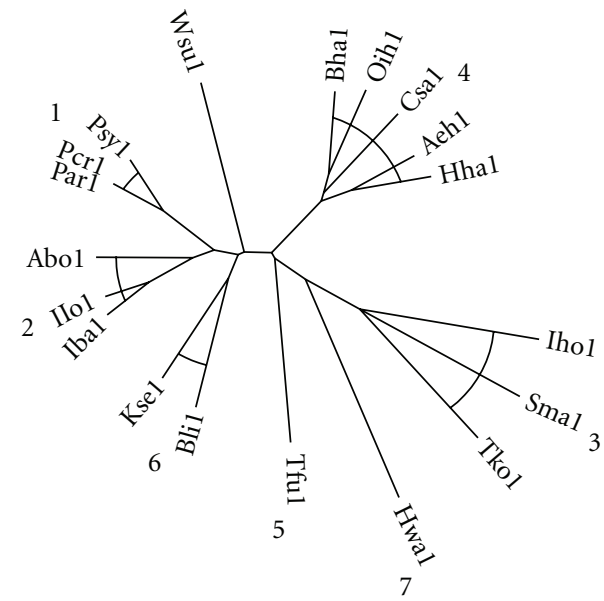

(a) CstA

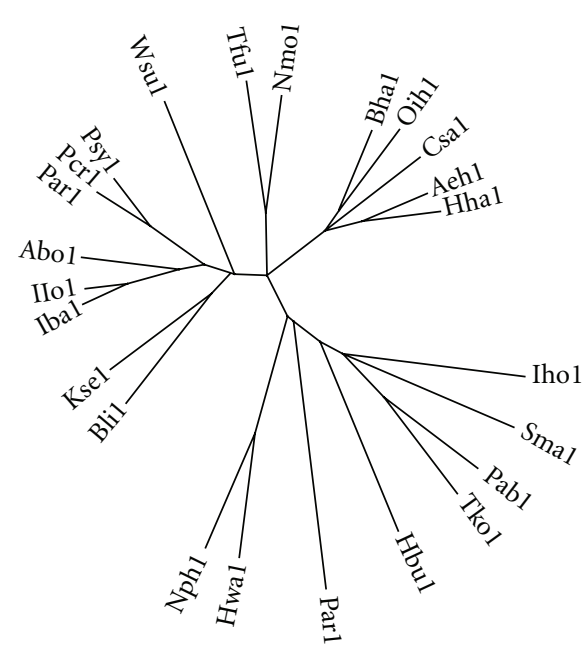

(c) CstA

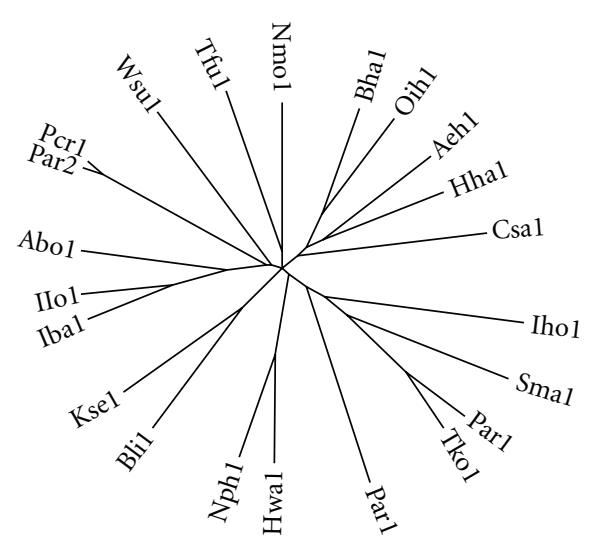

(e) ArsA

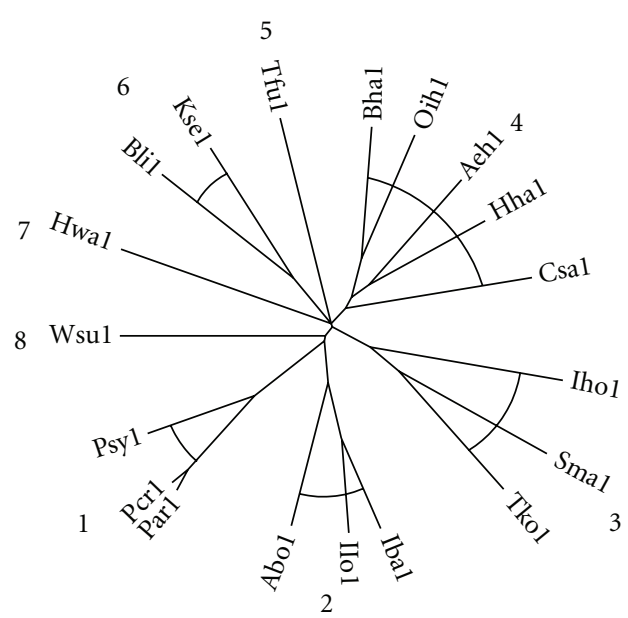

(b) ArsA

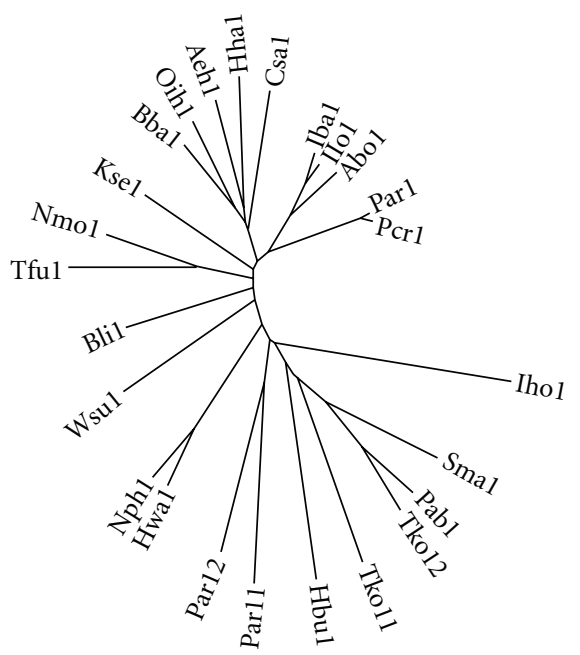

(d) CstX

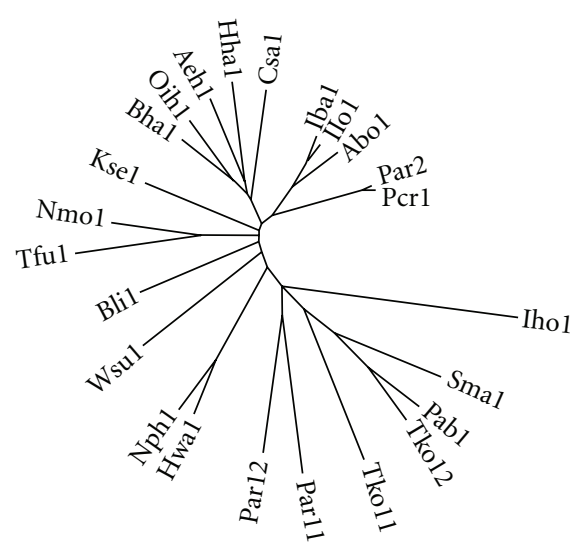

(f) CstX

Figure 2: Phylogenetic trees showing the coevolution of (a) CstA and (b) ArsA homologues, (c) CstA and (d) CstX homologues, and (e) ArsA and (f) CstX homologues, each pair being encoded within the same operons. The methodology was as described in Figure 1. Cluster numbers are assigned counterclockwise in (b) and retained in (a). Paralogues are distinguished with a second digit, either 1 or 2, in the protein abbreviations. 
functioning in oligomerization of the protein complex [31]. The GlpW protein (228 aas) is distantly related to the GvpL/F proteins. Finally, a BLAST search revealed that among the homologues of GvpK, one, of $\sim 158$ aas, possesses a Cterminal domain linked to an aspartyl nucleophile Haloacid Dehalogenase domain. Similar to the carbon starvation operons, the gas vesicle operons do not contain arsR.

3.4. ArsB Protein Associations. The phylogenetic trees for ArsA and ArsB (Figures 3(a) and 3(b), resp.) include proteins encoded in operons that include both homologues. These two trees reveal nearly identical clustering patterns. Thus, in both trees, there are three major branches, and within each cluster the corresponding proteins can be found. The Npu1 homologues branch distantly from the others, while the Sha and Sep proteins cluster tightly together. There are two Sha paralogues in the ArsB tree, suggesting that a recent gene duplication event only for these two proteins occurred for ArsB but not for ArsA. Examination of the operon encoding these paralogues revealed that the duplication encompassed not only the arsB gene but also the adjacent $\operatorname{ars} R$ and $\operatorname{ars} C$ genes which flank ars $B$. Similar intergenic spacing is observed for the two duplicated DNA segments. Finally, in the last cluster, we again see correspondence within the two trees. While Yin is distant from the other proteins, the Spu and She orthologues cluster tightly together as do the Yen and Eco proteins. The ArsB proteins in the third cluster are much more tightly clustered than are the ArsA proteins, suggesting that the latter have diverged in sequence more than the former.

ArsA/ArsB pairs not encoded within the same operon were added to the proteins represented in Figures 3(c) and 3(d). Examination of Figures S2(a) and S2(b) shows that the Sha1-3E and Sep1-2E ArsB paralogues may have coevolved with the ArsA homologues. However, these two ArsB homologues are clearly more divergent in sequence than the two other paralogues in this organism, Sha1-1 and Sha1-2. Therefore, we suggest that these two ArsB homologues have diverged in sequence more rapidly than their paralogues. We similarly examined the ArsB homologues Yen1-2E and Spu1-2E. In contrast to the results obtained with Sha1-3E and Sep1-2E, these paralogues appear to have arisen by a recent gene duplication event, and none of these paralogues have diverged in sequence more rapidly than the others. It is possible that these ArsB homologues are fully functional and that both sets of paralogues can be energized by the ArsA proteins. None of the other proteins in Figures S2(a) and S2(b) appear to have coevolved; (see Figures S2(a) and S2(b) in Supplementary Material available online at doi:10.1155/2010/187373).

3.5. Acr3 Protein Associations. By analyzing the Arsenic and Antimonite Resistance SEED subsystem spreadsheet, we identified 33 instances in 28 bacteria and archaea in which an ars operon contains an ars A homologue in a genome lacking an ars $B$ homologue. We also found 23 instances in 20 bacteria in which ars $A$ genes are located in an operon encoding Acr3 but not ArsB, and 9 instances in 9 bacteria where $\operatorname{acr} 3$ is in the same operon as ars $A$, but $\operatorname{ars} B$ and $\operatorname{ars} P$ are nowhere in the genome. These data indicate that some ArsA homologues from cluster 1 may function with Acr3, the only other characterized pump in the operon, as an ATPase energized As(III) efflux pump. These analyses suggest that these ArsA homologues may have functions independent of their role in the ArsAB translocation complex.

Trees for the ArsA and Acr3 proteins included in the same operons were derived as discussed above (Figures $3(\mathrm{c})$ and $3(\mathrm{~d})$, resp.). However, in contrast to the two examples described above, there appeared to be very little correspondence between these two trees. The only common clustering patterns were observed between Ahy1, Psyl, and Ppr1 which cluster similarly as do Bth1, Bth2, and Bvu1. However, this is where common clustering patterns end. In Figure 3(c), we find the former cluster loosely associated with proteins 7 through 11, while in Figure 3(d), these proteins cluster with proteins 4,5 , and 7. Moreover, proteins 8,9 , and 10 are together with proteins 6 and 7 in Figure 3(c), but they cluster with 20, 21, and 22 in Figure 3(d). Further examination of these two trees reveals that there are no additional similarities, clearly demonstrating that these two sets of proteins did not coevolve. The implication of this finding is that these proteins either do not function together, or that their associations do not depend on strict proteinprotein interactions. It should be noted that a lack of coevolution of proteins that interact physically to form a functional complex is rare but has been observed [32-34].

When examining acr3 and arsA pairs (Figures S2(c) and S2(d)) that map separately within the same genomes, we found very few phylogenetic correlations indicative of coevolution. However, several examples of late gene duplication were detected where the two paralogues showed high degrees of sequence identity. Within the ArsAs there are two examples, while in Acr3s three such paralogous pairs were identified.

3.6. ArsP Protein Associations. A previous study has noted an instance of an arsP gene, of unknown function, in an ars operon [5]. The Arsenic and Antimonite Resistance subsystem spreadsheet identified 61 instances, including the previously characterized ars $P$, in 52 bacteria and archaea where $\operatorname{ars} P$ is found in an ars operon. Our data demonstrate that genes coding for ArsP homologues are widely distributed in bacteria and archaea. In addition, there are two instances, one archaeal and one bacterial, in which ars $P$ is in the same operon as ars $A$ in the absence of either acr3 or ars $B$, tentatively suggesting a functional link between ArsA and ArsP. There are two distinct types of arsP, one encoding a $\sim 300$ aa 8 TMS putative transporter, another encoding a $\sim 400$ aa 8 TMS putative transporter with a $\sim 100$ aa hydrophilic insertion between the fourth and fifth TMSs. There are no clues as to the function(s) of ArsP homologues.

As described above, we constructed phylogenetic trees for the ArsA (Figure 3(e)) and ArsP (Figure 3(f)) homologues which coexist in operons. Comparison of these two trees reveals that clustering patterns are the same within experimental error with one exception. This exception refers to the presence of two distant Ame paralogues in the ArsP tree but 


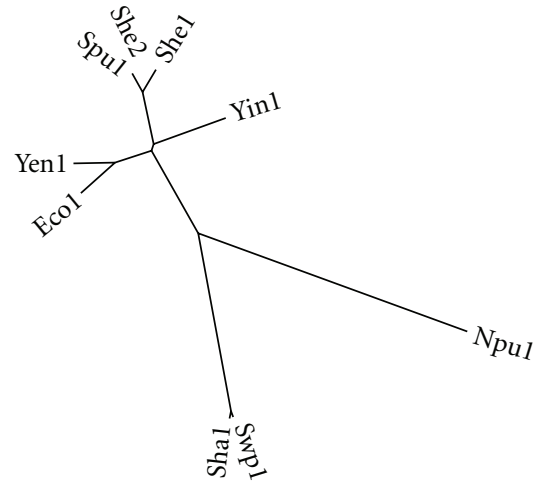

(a) ArsA

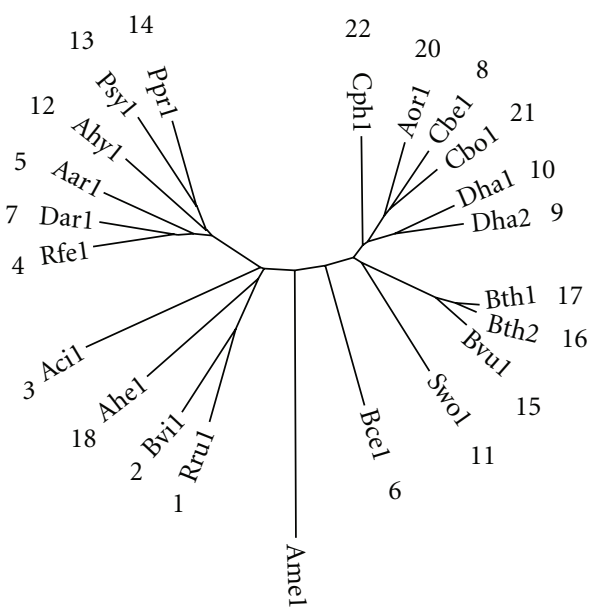

19

(c) ArsA

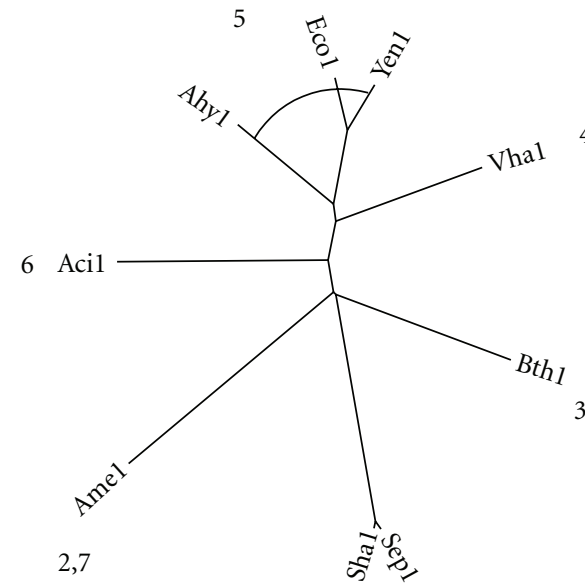

1

(e) ArsA

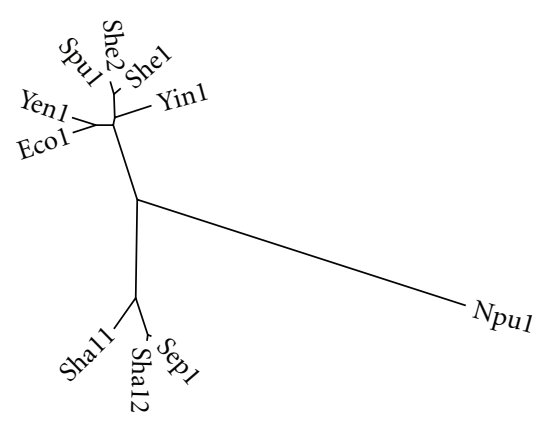

(b) ArsB

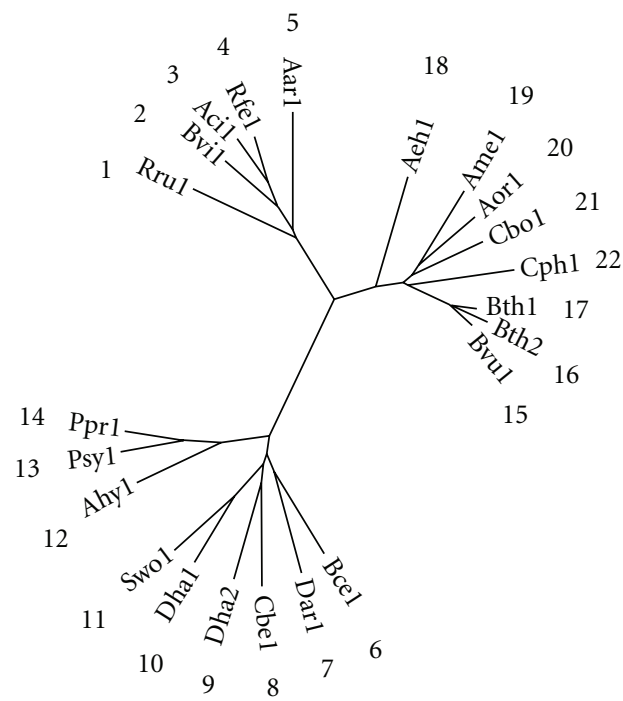

(d) Acr3

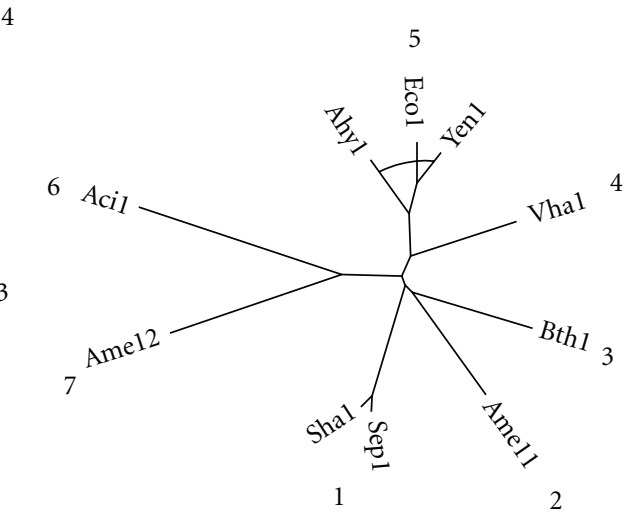

(f) $\operatorname{ArsP}$

Figure 3: Phylogenetic trees showing the coevolution of (a) ArsA and (b) ArsB homologues, (c) ArsA and (d) Acr3 homologues, and (e) ArsA and (f) ArsP homologues, each pair encoded within the same operons. Numbers are assigned in (d) and retained in (c). Numbers are assigned counterclockwise in (f) and retained in (e). Paralogues are distinguished with a second digit, either 1 or 2, in the protein abbreviations. gi numbers are provided in Table 1 . 
not in the ArsA tree. Excluding this paralogous arrangement, due to an extragenic duplication event, we find that the Sha and Sep proteins cluster tightly together (Cluster 1), that the Ahy, Eco, and Yen proteins cluster somewhat less tightly together (Cluster 5), and that branch 4 clusters loosely with Cluster 5 in both trees. Similarly, Clusters 1, 2, and 3 are more closely related to each other than they are to the other clusters on both trees. Finally, branch 6 is positioned between these two major clusters on both trees. The results show that ars $B$ homologues that occur within operons that also contain ars $P$ homologues coevolved. Overall, ArsP, ArsB, CstA, and CstX coevolved with different ArsA homologues, although Acr3 did not (see Section 4). It is interesting to note that within Cluster 1, the ArsP homologues are more distantly related to each other than are homologues in the ArsA tree, but that the opposite is true for all other corresponding pairs of proteins examined.

ArsA/ArsP pairs not encoded within the same operon were identified in the genomes of several organisms not represented in Figures 3(e) and 3(f). Examination of Figures S2(e) and S2(f) shows that Vfil ArsA and VfilE ArsP appear to have coevolved, as is also true for the clusters including Dha1E, Dha2E, and Aor1E. However, Spu1E ArsP, within the same cluster as Vhal and VfilE, does not appear to have coevolved with Spul ArsA. In fact, none of the other pairs of proteins in Figures S2(e) and S2(f) appear to have coevolved. It therefore seems unlikely that these proteins function together.

3.7. ArsD Protein Associations. The ArsD protein is a metallochaperone protein that is encoded in many ars operons that also encode ArsA. In fact, these two genes are frequently found adjacent to each other in these operons. We therefore conducted coevolutionary analyses of these two proteins as illustrated in Figures S3(a) and S3(b). Most of the 25 pairs of proteins showed similar clustering patterns, with a few exceptions. For example, Bcel clusters with Cbol, Cbe1, and Aor1 in the ArsD tree, but not in the ArsA tree. Furthermore, Gurl clusters with Aeh1 and Bvil in the ArsA tree but not in the ArsD tree. Branch lengths are comparable in the two trees, although some of the clusters such as the two large clusters at the top and bottom of the trees show that the branch lengths are shorter for the ArsA than the ArsD homologues, indicating that ArsA is less sequence divergent than ArsD. This, however, was not uniformly observed. Another difference was observed in the top cluster where in the ArsD tree, Psp1 and Ahy1 cluster loosely together and Vfil and Ppr1 cluster more tightly together. In contrast, in the ArsA tree, Psp1 clusters fairly tightly with Ppr1 and more loosely with Vfil, while Ahy1 occurs between the Asp1/Rfe1 cluster and Vfil. These differences appear too great to be accounted for by experimental error. In conclusion, it appears that ArsD and ArsA have coevolved in the majority of cases, but a significant fraction of these protein pairs showed divergence suggestive of horizontal gene transfer or nonuniform rates of sequence divergence. We suggest that these proteins did not always coevolve.
3.8. ArsH Protein Associations. Only four of the arsA containing operons in SEED proved to have ars $H$ genes. When the only four pairs of these proteins were examined from a phylogenetic standpoint, they showed no correspondence. For example, Yen1 and Kpn1 ArsH proteins cluster together but the corresponding ArsA proteins are on opposite sides of the ArsA tree. Although very few homologues were available for analysis, the results imply that ArsA and ArsH did not coevolve.

3.9. ars Operons Encoding $C c d A$, Thioredoxin, and RedoxActive Disulfide Protein 2. We have identified three new ars operon determinants, CcdA, thioredoxin, and redox-active disulfide protein 2 , coding for components of a putative arsenate reductase system. Using SEED, we found 8 instances in 7 organisms where these three redox genes are present in ars operons. In 7 of these 8 instances, ars $C$ genes are located in the operons with these determinants. CcdA is a DsbD homologue, which might catalyze electron transfer from the cytoplasm to the periplasm [37]. However, CcdA lacks the thioredoxin-like domain of the E. coli DsbD, suggesting that thioredoxin-like proteins present in the ars operons may donate electrons to CcdA. The redoxactive disulfide protein 2 has a thioredoxin-like conserved domain and is a disulfide oxidoreductase. The thioredoxinlike protein and redox-active disulfide protein 2 undoubtedly function in redox reactions, possibly where thioredoxinlike proteins transfer electron equivalents for thioredoxindependent ArsC reduction reactions [38].

3.10. The ArsA Superfamily. A Conserved Domain Search (CD-Search) of the NCBI protein database using the E. coli ArsA as the query sequence brought up a variety of ArsA-like ATPases with known associations. The AAA+ superfamily includes many proteins of different presumed physiological functions. Characterization of the AAA+ superfamily has been reported [39-41]. Our tree examines the AAA+ superfamily homologues of ArsA that are most closely related to the ArsA of E. coli. Based on NCBI descriptions of the conserved domains, (1) the FleN-like ATPase regulates motility by interacting with FleQ. (2) the ParA enzyme Caulobacter crescentus functions in chromosome segregation; when ADP binds, the protein interacts with single-stranded (ss) DNA, but when ATP is bound, ParB dissociates from its DNA binding sites. (3) CooC is a protein involved in maturation of the nickel center of carbon monoxide dehydrogenase. (4) The Fer4 or NifH protein uses ATP to facilitate the transfer of electrons to $\mathrm{N}_{2}$ or partially reduced forms of $\mathrm{N}_{2}\left(\mathrm{HN}=\mathrm{NH}\right.$ and $\left.\mathrm{H}_{2} \mathrm{~N}-\mathrm{NH}_{2}\right)$. NifH (component II) hydrolyzes ATP, energizing the transfer of electrons through an $\mathrm{Fe}_{4}-\mathrm{S}_{4}$ cluster to the other subunits within the nitrogenase complex. (5) MinD is a membraneassociated ATPase that regulates MinC and MinE, which function in the formation of the bacterial midcell septum. (6) BchL-like and ChlL proteins catalyze reductive formation of chlorophyllide. (7) An MRP-like ArsA homologue may be a component of an $\mathrm{Na}^{+} / \mathrm{H}^{+}$antiporter complex. (8) CbiA catalyzes the ATP-dependent amidation of various 
side chains of hydrogenobyrinic acid or cobyrinic acid a,c diamide in the synthesis of vitamin $B_{12}$. (9) DnaC is a DNA replication protein. (10) FlhG is an antiactivator of FleN. (11) Soj is an ATPase involved in chromosome partitioning in B. subtilis with homologues of the same function in many bacteria. (12) MipZ is an ATPase that forms a complex with the chromosome partitioning ParB and regulates FtsZ ring formation. (13) HslU is the ATPase component of the HslUV ATP-regulated protease/chaperone complex.

We investigated the relationships between the various ArsA homologues with CLUSTALX (Figure 4(a)), SuperfamilyTree1 (SFT1) (Figure 4(b)), and ProtPars (Figure 4(c)). CLUSTALX and SFT1 utilize neighbor-joining algorithms while ProtPars relies on parsimony. CLUSTALX and ProtPars base branching patterns on multiple alignments, but SFT1 uses BLAST bit scores [22]. Several patterns emerge when comparing the predicted evolutionary pathways taken by these families. In all three trees we find that Clusters 10 (Mrp) and 11 (MinD, FlhG, and FleN-like) are together, Clusters 1 (HslU), 4 (CbiA), and 5 (Fer4_NifH) are together, and Clusters 7 (Soj) and 8 (ParA) are together. Clusters 9 (BchX, ChlL, NifH, and Bch1-like), 10 (MRP), 11 (MinD, FlhG, and FleN-like), and 12 (CooC) are found together in trees A and C. Clusters 1 (HslU), 3 (DnaC), 4 (CbiA), and 5 (Fer4_NifH) are found together in trees A and B. Finally, Clusters 12 (CooC) and 13 (ArsA) are found together in trees $\mathrm{A}$ and $\mathrm{B}$. These results provide information about the relative phylogenetic distances for these AAA+ ATPases, thus suggesting their functional/mechanistic relationships.

\subsection{Orthologous Relationships Based on Comparison with} $16 S$ and $18 S$ rRNA Phylogeny. The ribosomal RNA (rRNA) tree (Figure 5) for the organisms included in this study exhibits clustering patterns pretty much as expected with the different phyla grouping separately. Of note, the one representative $\mathcal{\varepsilon}$-proteobacterial RNA clusters loosely with the Bacteroidetes homologues instead of the large proteobacterial RNA cluster.

As indicated in Table 1, Cluster 1 of Figure 4 includes proteins derived from proteobacteria and other phyla, each clustering together, but with many exceptions. For example, Bth1 (Bacteroidetes) is sandwiched in between Firmicute homologues. Clustering together in the following order are Gur1 ( $\delta$-Proteobacterium), Bph1 ( $\beta$-Proteobacterium), Mmal, and Rru1 ( $\alpha$-Proteobacteria). Additionally, we find two proteins from Verrucomicrobia and Planctomycetes, respectively, clustering together with these proteobacterial proteins. Also, among a group of proteobacterial proteins, we find one homologue from Chlorobi and three from Actinobacteria. Euryarchaeata proteins cluster separately at the base of Cluster 1 as well as in Cluster 2. These results clearly demonstrate that there has been extensive horizontal ars $A$ gene transfer during the evolution of these organisms.

Cluster 3 proteins are derived exclusively from eukaryotes. However, many different phyla are represented. Progressing in the clockwise direction, the first two proteins, which group together, are from Trichomonada species. Then we find a cluster of fungal proteins with two deeply rooted members, Call and Sce1, possible examples of horizontal gene transfer. Tad1 is a metazoan protein which clusters tightly together with four-plant proteins. This also appears to be an example of horizontal gene transfer since all other Metazoan proteins cluster separately. Remaining proteins in Cluster 3 are relatively distantly related to each other and are derived from four distinct phyla. Since proteins from each phylum cluster together, it is reasonable to suggest that these were inherited by vertical descent.

Cluster 4 consists of proteins derived from Cyanobacteria with one exception, a $\delta$-proteobacterial protein. This last protein is more distantly related to the cyanobacterial proteins than they are to each other. Further, the protein and rRNA trees for Cyanobacteria are in agreement. Therefore, the results are consistent with orthology for all of these proteins. It should be noted that genome context analyses indicated that these proteins are associated with gas vesicle biogenesis.

Cluster 5 consists exclusively of plant and algal proteins. While much of the evidence suggests orthology, it should be noted that Arabidopsis, Vitis, and Zea all have two paralogues which, however, do not exhibit orthologous relationships between them. It seems likely that these proteins arose by more than one gene duplication event. Cluster 6 consists only of two distantly related proteins from two different phyla, while Cluster 7 consists of archaeal proteins with phylogenetic relationships consistent with orthology. These proteins may function together with CstA in carbon stress responses.

Cluster 8 includes proteins from eight different bacterial phyla. Moreover, the Chloroflexi and Chlorobi proteins each fall into three distinct subclusters. The two $\delta$-proteobacterial proteins are also distantly related. The results are consistent either with horizontal gene transfer or with early gene duplication events.

Cluster 9 includes proteins from four different bacterial and archaeal phyla. These clearly do not exhibit orthology, but as for Cluster 8, the basis for this observation is not clear. Cluster 10 consists of proteins only from Actinobacteria, and with only one protein derived from any one genus; the results are fully consistent with orthology (compare Figures 1 and 5). Cluster 11 consists of only four proteins derived from three different phyla. Orthology seems unlikely. Clusters 12 and 13 each consists of two distantly related proteins from two different phyla. We are hesitant to predict orthology for these proteins. Cluster 14 consists of six proteins. Two distant members derive from Crenarchaeota, and four close members derive from Euryarchaeota. The results are consistent with orthology. Cluster 15 consists of four proteins two from archaea and two from bacteria. Surprisingly, each of the bacterial proteins clusters together with an archaeal protein, and the phylogenetic distance is the same for these two pairs. The basis for these relationships is unknown, but horizontal transfer probably played a role.

The three proteins in Cluster 16 exhibit relationships potentially consistent with orthology. Cluster 17 consists of twelve proteins from three bacterial phyla and one archaeal phylum. The $\gamma$-proteobacterial proteins fall into three distinct clusters, inconsistent with orthology. The 


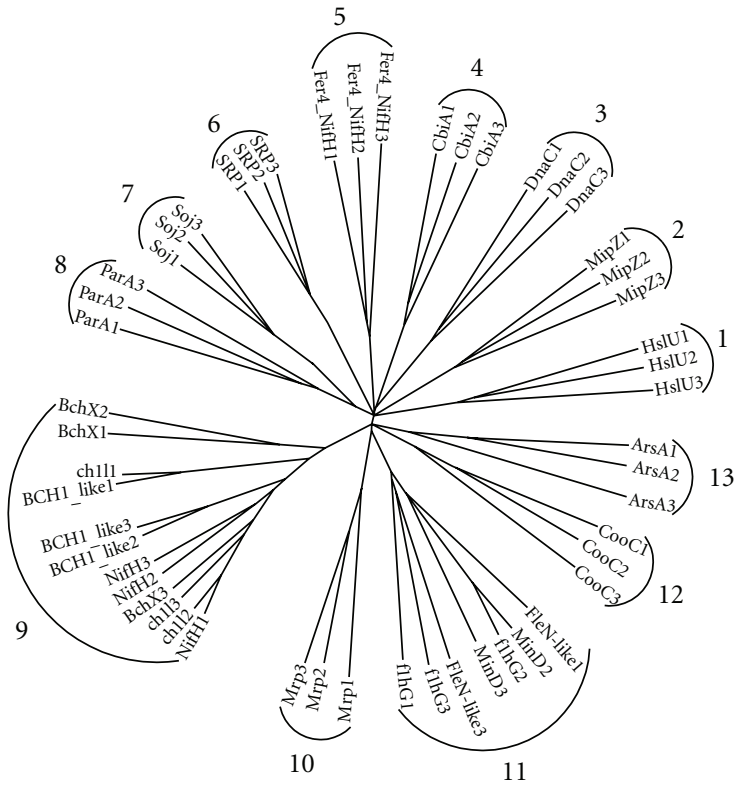

(a)

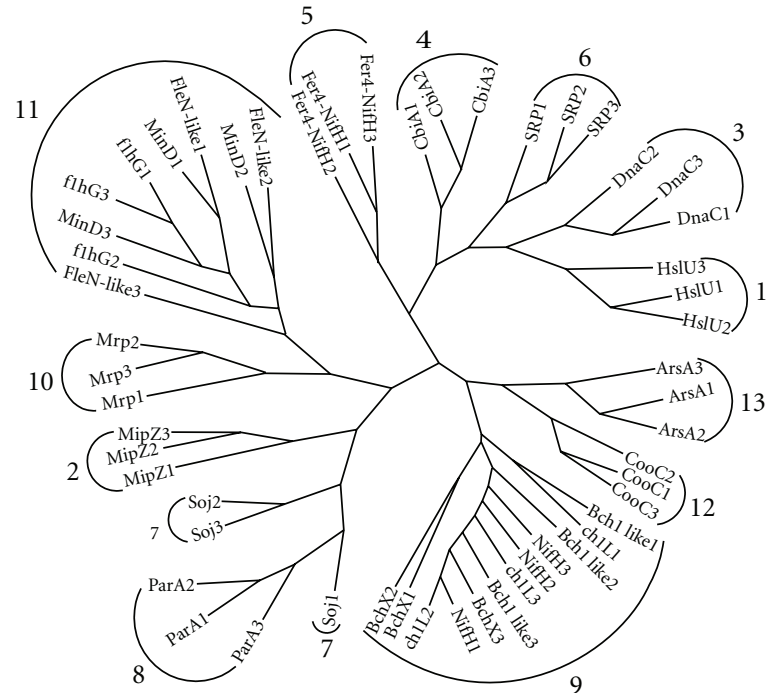

(b)

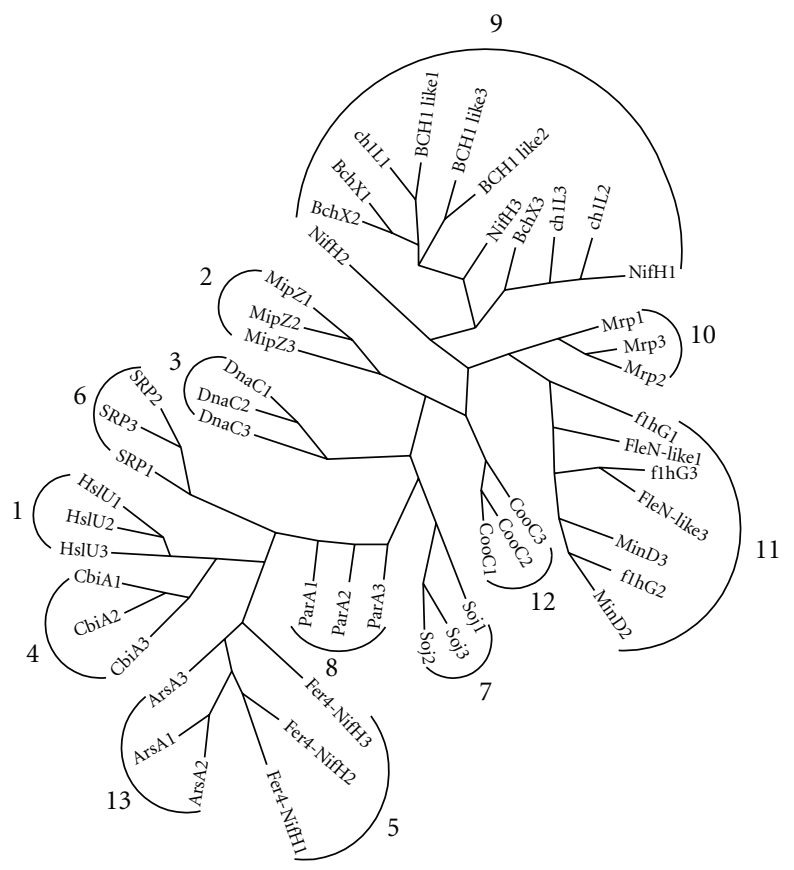

(c)

FIGURE 4: (a) CLUSTALX multiple alignment showing the relationships of the representative proteins to each other. Representative proteins from each family were selected following an NCBI BLASTP search, selecting sequences with $30 \%-50 \%$ identity and an $e$-value less than $e^{-60}$. (b) SuperfamilyTree created with representative proteins using the SFT1 program [22] without a multiple alignment and using TreeView [24]. (c) Representative proteins were analyzed, and the tree was generated using ProtPars [25]. Both CLUSTALX and SFT1 use neighborjoining algorithms to generate the trees while ProtPars uses parsimony. These trees show the evolutionary relationships of ArsA homologues to each other. Numbers are assigned in (a) and retained in (b) and (c).

four archaeal proteins cluster separately from the bacterial proteins, and form relationships suggesting that only the Haloarcular homologue may not be orthologous to the others. It seems unlikely that the members of Cluster 18 are orthologous.
In summary, most of the 18 clusters consist of proteins that are not likely to be orthologous although a few exceptions have been noted. While horizontal gene transfer appears to account for much of this divergence, we consider it equally likely that early gene duplication events, giving rise to multiple paralogues, occurred within this protein family. 


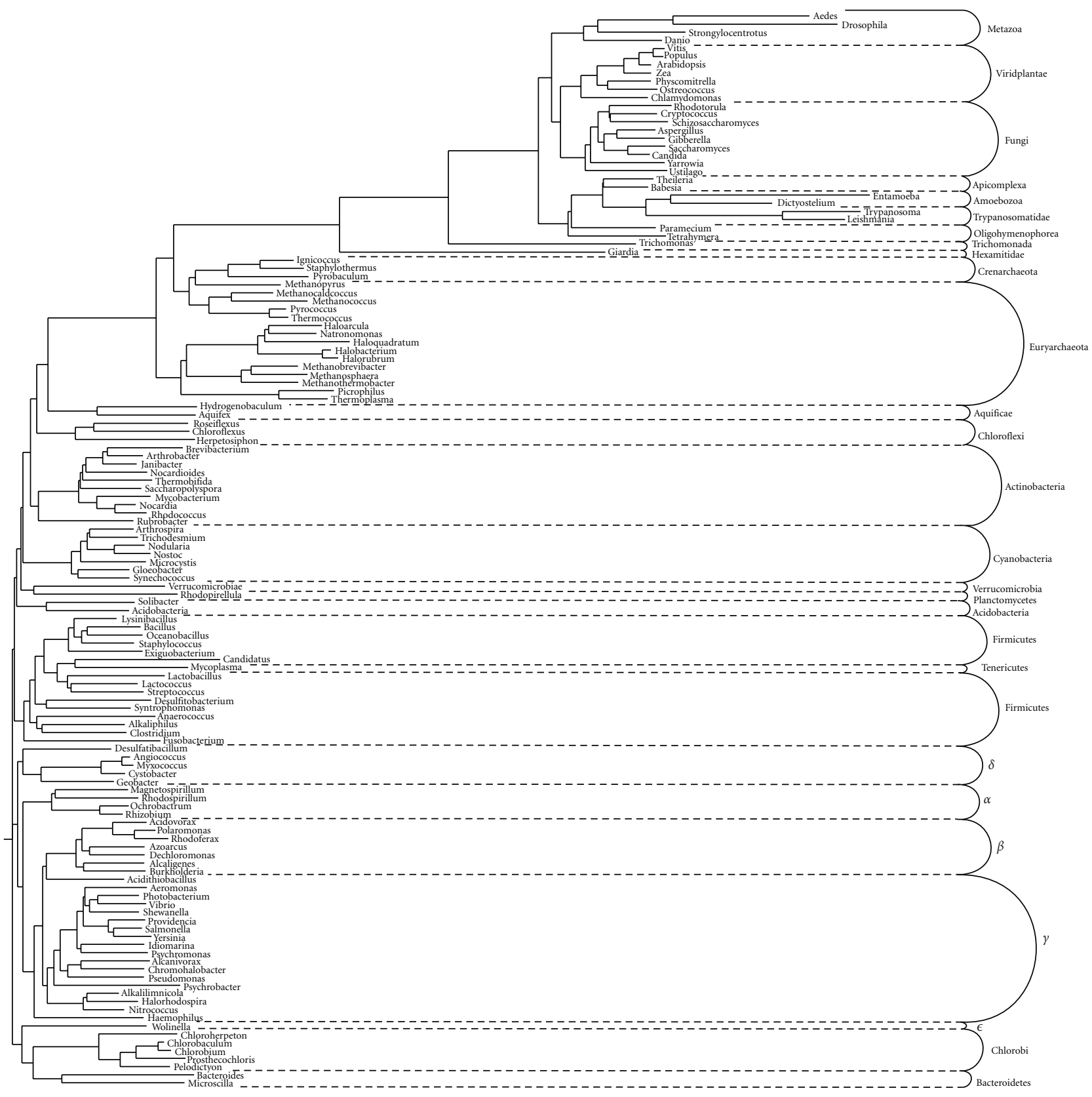

FIGURE 5: Phylogenetic tree of $16 \mathrm{~S}$ and $18 \mathrm{~S}$ rRNA nucleotide sequences of genera represented in this study. The methodology was as described in Figure 1. Sequences were derived from the NCBI nucleotide database. Clusters correlate with organismal type.

3.12. Conserved Motifs in ArsA Homologues. The MEME program [30] was used to examine the occurrence of conserved motifs within the 112 ArsA homologues included in the training set, 80 single domain and 32 double domain proteins. The two most conserved motifs obtained with this program are shown in Figure 6. The best conserved motif (motif 1 in Figure 6) includes the Rossmann fold motif (GXGXXG) which is fully conserved at the beginning of this motif. The sequence of this fully conserved motif (positions 1-7) plus the well-conserved adjacent residues at positions $8-13$ is GKGGVGK(T/S $)_{2} \mathrm{X}(\mathrm{S} / \mathrm{A})(\mathrm{A} / \mathrm{C} / \mathrm{S})(\mathrm{A} / \mathrm{S})$ [alternative residues at a single position are in parentheses]. Additionally, at positions 29-33, we found another well-conserved submotif $(\mathrm{S} / \mathrm{T})(\mathrm{T} / \mathrm{S}) \mathrm{DPA}$ where the only substitutions are in the $\mathrm{P}$ which is replaced by an $\mathrm{A}, \mathrm{T}$, or $\mathrm{M}$ in just four of the fifty homologues included in this analysis. As expected, the single domain proteins had a single such motif while the double domain proteins had two such motifs which differed from each other in no significant way.

The second best conserved motif is the 12-residue motif (DTAPTGHTIRLL), called the DTAP motif [9]. The DTAP motif appears to be a conformationally flexible 


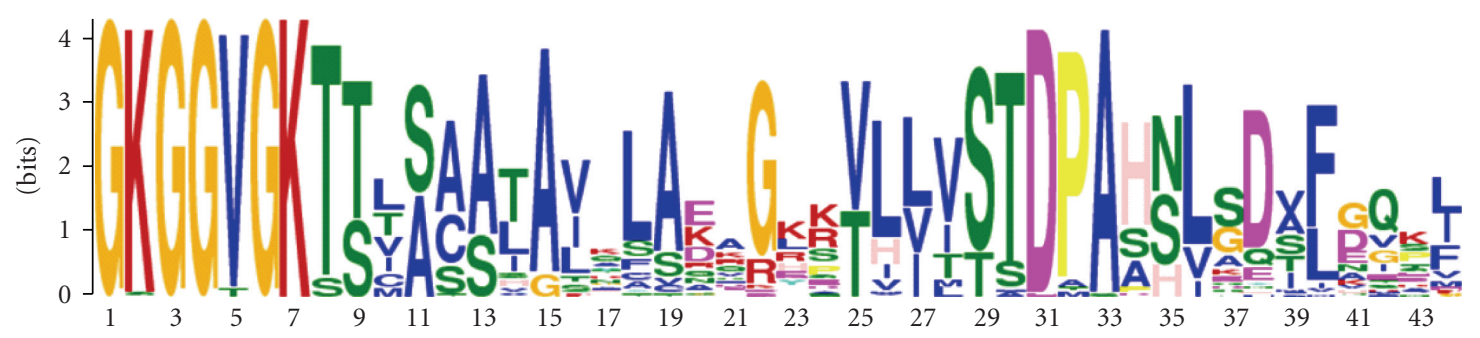

(a)

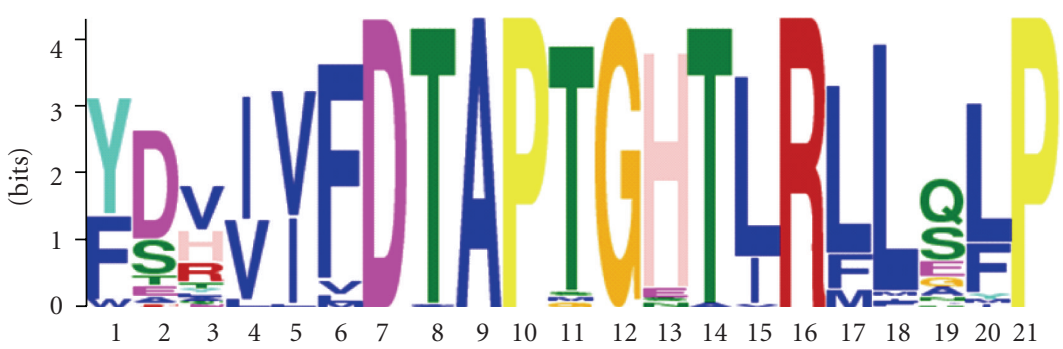

(b)

FIGURE 6: MEME [30] sequence logos illustrating the conserved residues of (a) motif 1 and (b) motif 2 found in ArsA homologues. The size of the letters indicates amino acid conservation, with larger letters representing more conserved residues.

region which facilitates the interconversion of the ATP/ADP substrate/product binding site(s). This exceptionally wellconserved motif corresponds precisely to that reported by Rensing et al. [9]. Additionally, following the Rossmann fold motif, an SXD motif is observed with the S and D almost fully conserved. Finally, towards the C-terminus of these proteins is a well-conserved $\mathrm{N}$ residue that is fully conserved in the single domain proteins (see below).

Three cysteine residues have been shown to be important for the activity of the E. coli ArsA protein, C113, C172, and C422 [9]. The conservation of these cysteines was examined separately in multiple alignments of both the double and the single domain proteins. Double domain proteins can be found in Clusters 1, 4, and 6, while all remaining clusters include proteins with only a single domain. None of these three cysteine residues were conserved in the single domain proteins. In all Cluster 1 proteins, the $\mathrm{C}$ is fully conserved with a single exception: C172 is replaced by an $\mathrm{N}$ in Sty1. However, in Clusters 4 and 6, none of these cysteines are well conserved. In fact, a W is found in all but one (Gur2) of the Cluster 4 proteins, where an $\mathrm{F}$ can be found, for $\mathrm{C} 113$, while an $\mathrm{L}$ and a $\mathrm{V}$ replace the $\mathrm{C}$ residue in the two cluster 6 proteins. C172 is replaced by an S or T in Cluster 4 proteins except for Gur2 where an $\mathrm{R}$ is found. $\mathrm{V}$ and $\mathrm{K}$ residues replace this $C$ in the two Cluster 6 proteins. $C 422$ is fully conserved in Cluster 1 proteins, but in Cluster 4 homologues, an aliphatic hydrophobic residue always occurs. Finally, in the two Cluster 6 proteins a G and an L occur. It seems clear from this analysis that the mechanism of action of Cluster 1 homologues differs substantially from that of all other proteins in the ArsA tree (Figure 1).

The single domain proteins were separately aligned, and they were found to have a well-conserved Rossmann fold motif GKGG(V/T)GK(T/S). The DTAP motif could also be recognized, but it was less well conserved than for the double domain proteins. Additionally, the three well-conserved cysteines present in the double domain proteins and essential for activity for the $E$. coli protein were not conserved in any of the single domain proteins. However, two regions in these single domain proteins proved to be well conserved. These were the SXD motif in the beginning of the proteins, where the $\mathrm{S}$ is fully conserved and the $\mathrm{D}$ can be substituted only by $\mathrm{N}$, and the fully conserved $\mathrm{C}$-terminal $\mathrm{N}$ residue mentioned above. Thus, it is clear that the mechanisms of action must be quite different, but these differences distribute largely according to phylogenetic clusters.

\section{Discussion}

The apparent functional promiscuity of ArsA homologues is worthy of note. Our phylogenetic analyses have provided evidence for the coevolution of different sets of ArsA homologues with (1) ArsB, ArsP, and frequently ArsD, (2) CstA and CstX, and (3) gas vesicle biogenesis proteins. However, ArsA and Acr3 homologues did not coevolve, and the few ArsH homologues identified also appeared to have evolved independently of ArsA. Nevertheless, we have demonstrated that $\operatorname{ars} B$, ars $P$, ars $D$, and $\operatorname{acr} 3$ often occur within the same operons as ars $A$ homologues; $\operatorname{cst} A, \operatorname{cst} X$, and $\operatorname{cst} Y$ are also found in operons with $\operatorname{ars} A$ homologues, and CstA "looks" like a permease. Coevolution and coclustering of the encoding genes suggest common functions and mutual interactions.

To support the hypothesis that ArsA homologues function in conjunction with these various permeases, we have examined instances in which an ars A homologue is found in 
an operon containing one of the four putative transporters mentioned above in the absence of the other three. We have identified nine such instances for Acr3, eleven for ArsP, ten for ArsB, and 26 for CstA. Strikingly, none of the arsenic resistance genes are found in operons with carbon starvation genes. The converse is true as well, clearly suggesting a function in arsenic resistance for the former gene sets, but not for the latter.

Based on phylogenetic trees, we suggest that ArsA has coevolved with the above proteins with the notable exception of Acr3. We therefore hypothesize that ArsA homologues function not only with ArsB but also, in specific instances, with ArsP and CstA. Analysis of potential coevolving protein pairs, for proteins not present within the same operons or gene clusters, revealed a few cases of possible coevolution, but in many cases, coevolution seemed impossible.

It should be noted that although Acr3 may not have coevolved with ArsA homologues, acr3 genes are found nine times within operons also containing ars A homologues but lacking $\operatorname{ars} B$ and $\operatorname{ars} P$ genes anywhere in the genomes. There are 23 instances in which acr 3 genes are found with an $\operatorname{ars} A$ homologue in an operon without an arsB. Thus, ArsA homologues may either function with an unidentified protein not found in the operon or with the cotranscribed acr3 homologue. A recent study has proposed, based upon biochemical analyses, that ArsA homologues do indeed function with Acr3 [17]. A cloned A. metalliredigens arsa1 operon, contains two single domain ars $A$ homologues and an acr3 homologue. When transferred to E. coli, increased resistance to arsenite was observed when the ars $A$ and acr 3 homologues were expressed together. In contrast, no such increased resistance was observed when the acr 3 homologue was expressed alone [17]. Given the widespread colocalization of ars $A$ and $\operatorname{acr} 3$ homologues and the recent biochemical analyses of $\mathrm{Fu}$ et al. [17], we agree that ArsA is likely to energize Acr3-mediated arsenite efflux.

The case of Outer Membrane Pore-Forming Factors (OMFs) is particularly relevant to this discussion. OMF proteins function with various cytoplasmic membrane transport systems but did not coevolve with these systems [34]. An explanation might be promiscuity with respect to the protein-protein interactions required for function. It is known, for example, that a single OMF (e.g., TolC of E. coli) can function with several different transporters, although the cofunctioning Membrane Fusion Proteins (MFPs) do not (see TCDB). Thus, proteins that exhibit a lack of coevolution can still function together, but the physical association may be less stringent.

Conserved motifs in ArsA homologues were examined using the MEME program [30]. Concerning the internally duplicated ArsA homologues of phylogenetic clusters 1, 4, and 6, the Rossmann fold and surrounding regions proved to be almost identical, and consequently only the motif for the first halves, together with all single domain homologues, was presented. The Rossmann fold was fully conserved in virtually all homologues, suggesting that none of the proteins included in our study were the products of pseudogenes. Additionally, the DTAP domain, thought to facilitate the interconversion of the ATP/ADP substrate/product binding site(s), also proved to be remarkably well conserved among all of the homologues examined. In fact, conservation in both motifs was more extensive than previously recognized, suggesting that additional residues will prove to be crucial for function. It is also worthy of note that the exceptionally well-conserved $\mathrm{N}$-terminal SXD and C-terminal $\mathrm{N}$ residues are also present in the two domain proteins, as well as all homologues, again suggesting that these residues play a role in the basic mechanism used by these proteins.

The remarkable conservation of function noted above contrasts with the lack of conservation of the three cysteine residues, which in the E. coli ArsA protein have been shown to be essential for function [9]. These residues were fully conserved in Cluster 1, which includes this double domain E. coli protein. In fact, all members of this cluster appear to have the same general function and catalyze ATP hydrolysis, coupled to transport by the same mechanism. Of even greater interest is the conclusion that none of the other homologues can function by the same mechanism since they lack these cysteines. These analyses thus indicate not only that the functions of these proteins will differ, but also that their mechanisms of action will differ.

We have found that some homologues of ars $A$ are found in operons encoding gas vesicle biogenesis proteins. It can be assumed that the ATPase functions to energize a biogenic step. Since GvpN also appears to be an ATPase, it seems reasonable to propose that these two proteins function to energize distinct but related functions. The Hsp20 protein, also often present in gas vesicle gene clusters, presumably plays a chaperone role in protein complex formation.

By examining the ArsA homologue phylogenetic tree, we found that genes encoding Cluster 1 homologues are associated with $\operatorname{ars} B$, ars $P$, and $\operatorname{acr} 3$, Cluster 4 and 6 homologues are encoded together with gas vesicle biogenesis proteins, and cluster 14-18 homologues are found with carbon starvation genes cst $A, \operatorname{cst} X$, and sometimes, cst $Y$. CstA possibly functions in peptide uptake [42]; CstX might couple CstA-catalyzed transport to ArsA-dependent ATP hydrolysis and is never found encoded in an operon without cstA. This fact strongly suggests a close functional and physical relationship between these two gene products. CstY is a protein found in five archaea, encoded within operons together with $\operatorname{cst} A$ and $c s t X$. CstY might well provide a nonessential auxiliary function. Unlike the ars operons, the gas vesicle and carbon starvation operons are probably not controlled by arsenite concentrations because they do not contain associated arsR genes.

ArsA homologues associated with arsenic resistance and gas vesicle biogenesis have two domains and are $\sim 600$ aas long. In contrast, ArsA homologues associated with carbon starvation genes have one domain and are $~ 300$ aas long. Why the internally duplicated ArsAs are always associated with arsenic resistance or $g v p$ genes while those that function with carbon starvation proteins possess just one ArsA domain is unknown. The latter ArsAs may function as homodimers. We have also found 60 new instances of ArsP homologues based on their association with other ars operon determinants. Although the function of ArsP homologues is 
unknown, they might play a backup role in As(III) export or act on a related substrate.

We have identified three putative oxidoreduction genes present in ars operons. They encode homologues of CcdA, thioredoxin, and redox-active disulfide protein 2 which may function with ArsC, the $\mathrm{As}(\mathrm{V})$ reductase. Thioredoxin is likely to provide electrons to ArsC, and CcdA might transfer electrons across the membrane as does DsbD. However, not all DsbD homologues appear to catalyze transmembrane electron flow. A function in transmembrane electron flow seems less likely since ArsC is cytoplasmic. Clearly the reported studies open up many avenues of molecular genetic inquiry.

\section{Acknowledgments}

The authors wish to thank Barry Rosen for the valuable suggestions and criticisms, Andrei Osterman for the helpful discussion and Lesya Castillo for the assistance with figures. This paper was supported by NIH Grant GM077402.

\section{References}

[1] R. S. Oremland and J. F. Stolz, "Arsenic, microbes and contaminated aquifers," Trends in Microbiology, vol. 13, no. 2, pp. 45-49, 2005.

[2] C. R. Jackson and S. L. Dugas, "Phylogenetic analysis of bacterial and archaeal arsC gene sequences suggests an ancient, common origin for arsenate reductase," BMC Evolutionary Biology, vol. 3, article no. 18, 2003.

[3] Y.-F. Lin, A. R. Walmsley, and B. P. Rosen, "An arsenic metallochaperone for an arsenic detoxification pump," Proceedings of the National Academy of Sciences of the United States of America, vol. 103, no. 42, pp. 15617-15622, 2006.

[4] I. I. Vorontsov, G. Minasov, J. S. Brunzelle et al., "Crystal structure of an apo form of Shigella flexneri ArsH protein with an NADPH-dependent FMN reductase activity," Protein Science, vol. 16, no. 11, pp. 2483-2490, 2007.

[5] L. Wang, B. Jeon, O. Sahin, and Q. Zhang, "Identification of an arsenic resistance and arsenic-sensing system in Campylobacter jejuni," Applied and Environmental Microbiology, vol. 75, no. 15, pp. 5064-5073, 2009.

[6] L. S. Tisa and B. P. Rosen, "Molecular characterization of an anion pump. The ArsB protein is the membrane anchor for the ArsA protein," Journal of Biological Chemistry, vol. 265, no. 1, pp. 190-194, 1990.

[7] S. Dey, D. Dou, L. S. Tisa, and B. P. Rosen, "Interaction of the catalytic and the membrane subunits of an oxyaniontranslocating ATPase," Archives of Biochemistry and Biophysics, vol. 311, no. 2, pp. 418-424, 1994.

[8] B. P. Rosen, H. Bhattacharjee, T. Zhou, and A. R. Walmsley, "Mechanism of the ArsA ATPase," Biochimica et Biophysica Acta, vol. 1461, no. 2, pp. 207-215, 1999.

[9] C. Rensing, M. Ghosh, and B. P. Rosen, "Families of softmetal-ion-transporting ATPases," Journal of Bacteriology, vol. 181, no. 19, pp. 5891-5897, 1999.

[10] G. Kao, C. Nordenson, M. Still, A. Rönnlund, S. Tuck, and P. Naredi, "ASNA-1 positively regulates insulin secretion in $C$. elegans and mammalian cells," Cell, vol. 128, no. 3, pp. 577587, 2007.
[11] B. Kurdi-Haidar, D. Heath, S. Aebi, and S. B. Howell, "Biochemical characterization of the human arsenite-stimulated ATPase (hASNA-I)," Journal of Biological Chemistry, vol. 273, no. 35, pp. 22173-22176, 1998.

[12] J. Metz, A. Wächter, B. Schmidt, J. M. Bujnicki, and B. Schwappach, "The yeast Arr4p ATPase binds the chloride transporter Geflp when copper is available in the cytosol," Journal of Biological Chemistry, vol. 281, no. 1, pp. 410-417, 2006.

[13] J. Shen, C.-M. Hsu, B.-K. Kang, B. P. Rosen, and H. Bhattacharjee, "The Saccharomyces cerevisiae Arr4p is involved in metal and heat tolerance," BioMetals, vol. 16, no. 3, pp. 369378, 2003.

[14] S. Stefanovic and R. S. Hegde, "Identification of a targeting factor for posttranslational membrane protein insertion into the ER," Cell, vol. 128, no. 6, pp. 1147-1159, 2007.

[15] Y.-Y. Tseng, C.-W. Yu, and V. H.-C. Liao, "Caenorhabditis elegans expresses a functional ArsA,” FEBS Journal, vol. 274, no. 10, pp. 2566-2572, 2007.

[16] M. H. Saier Jr., "Protein secretion and membrane insertion systems in gram-negative bacteria," Journal of Membrane Biology, vol. 214, no. 1-2, pp. 75-90, 2006.

[17] H. -L. Fu, B. P. Rosen, and H. Bhattacharjee, "Biochemical characterization of a novel ArsA ATPase complex from Alkaliphilus metalliredigens QYMF," FEBS Letters, vol. 584, no. 14, pp. 3089-3094, 2010.

[18] W. Busch and M. H. Saier Jr., "The Transporter Classification (TC) system, 2002," Critical Reviews in Biochemistry and Molecular Biology, vol. 37, no. 5, pp. 287-337, 2002.

[19] M. H. Saier Jr., C. V. Tran, and R. D. Barabote, “TCDB: the Transporter Classification Database for membrane transport protein analyses and information," Nucleic acids research., vol. 34, pp. D181-D186, 2006.

[20] M. H. Saier Jr., M. R. Yen, K. Noto, D. G. Tamang, and C. Elkan, "The Transporter Classification Database: recent advances," Nucleic Acids Research, vol. 37, no. 1, pp. D274D278, 2009.

[21] S. F. Altschul and E. V. Koonin, "Iterated profile searches with PSI-BLAST - a tool for discovery in protein databases," Trends in Biochemical Sciences, vol. 23, no. 11, pp. 444-447, 1998.

[22] M. R. Yen, J. Choi, and M. H. Saier Jr., "Bioinformatic analyses of transmembrane transport: novel software for deducing protein phylogeny, topology, and evolution," Journal of Molecular Microbiology and Biotechnology, vol. 17, no. 4, pp. 163-176, 2009.

[23] J. D. Thompson, T. J. Gibson, F. Plewniak, F. Jeanmougin, and D. G. Higgins, "The CLUSTAL X windows interface: flexible strategies for multiple sequence alignment aided by quality analysis tools," Nucleic Acids Research, vol. 25, no. 24, pp. 4876-4882, 1997.

[24] Y. Zhai, J. Tchieu, and M. H. Saier Jr., "A web-based Tree View (TV) program for the visualization of phylogenetic trees," Journal of Molecular Microbiology and Biotechnology, vol. 4, no. 1, pp. 69-70, 2002.

[25] J. Felsenstein, "PHYLIP_Phylogeny Inference Package (Version 3.2)," Cladistics, vol. 5, pp. 164-166, 1989.

[26] Y. Zhai and M. H. Saier Jr., "A web-based program (WHAT) for the simultaneous prediction of hydropathy, amphipathicity, secondary structure and transmembrane topology for a single protein sequence," Journal of Molecular Microbiology and Biotechnology, vol. 3, no. 4, pp. 501-502, 2001. 
[27] Y. Zhai and M. H. Saier Jr., "A web-based program for the prediction of average hydropathy, average amphipathicity and average similarity of multiply aligned homologous proteins," Journal of Molecular Microbiology and Biotechnology, vol. 3, no. 2, pp. 285-286, 2001.

[28] R. Overbeek, T. Begley, R. M. Butler et al., "The subsystems approach to genome annotation and its use in the project to annotate 1000 genomes," Nucleic Acids Research, vol. 33, no. 17, pp. 5691-5702, 2005.

[29] D. A. Rodionov, J. De Ingeniis, C. Mancini et al., "Transcriptional regulation of NAD metabolism in bacteria: NrtR family of Nudix-related regulators," Nucleic Acids Research, vol. 36, no. 6, pp. 2047-2059, 2008.

[30] T. L. Bailey and C. Elkan, "The value of prior knowledge in discovering motifs with MEME," in Proceedings of the International Conference on Intelligent Systems for Molecular Biology (ISMB '95), vol. 3, pp. 21-29, 1995.

[31] H. D. Shukla and S. DasSarma, "Complexity of gas vesicle biogenesis in Halobacterium sp. strain NRC-1: identification of five new proteins," Journal of Bacteriology, vol. 186, no. 10, pp. 3182-3186, 2004.

[32] S. Broer, G. Ji, A. Broer, and S. Silver, "Arsenic efflux governed by the arsenic resistance determinant of Staphylococcus aureus plasmid pI258," Journal of Bacteriology, vol. 175, no. 11, pp. 3480-3485, 1993.

[33] D. Dou, S. Dey, and B. P. Rosen, "A functional chimeric membrane subunit of an ion-translocating ATPase," Antonie van Leeuwenhoek, International Journal of General and Molecular Microbiology, vol. 65, no. 4, pp. 359-368, 1994.

[34] I. T. Paulsen, J. H. Park, P. S. Choi, and M. H. Saier Jr., "A family of Gram-negative bacterial outer membrane factors that function in the export of proteins, carbohydrates, drugs and heavy metals from Gram-negative bacteria," FEMS Microbiology Letters, vol. 156, no. 1, pp. 1-8, 1997.

[35] G. van Keulen, D. A. Hopwood, L. Dijkhuizen, and R. G. Sawers, "Gas vesicles in actinomycetes: old buoys in novel habitats?" Trends in Microbiology, vol. 13, no. 8, pp. 350-354, 2005.

[36] A. Mlouka, K. Comte, A.-M. Castets, C. Bouchier, and N. Tandeau De Marsac, "The gas vesicle gene cluster from Microcystis aeruginosa and DNA rearrangements that lead to loss of cell buoyancy," Journal of Bacteriology, vol. 186, no. 8, pp. 2355-2365, 2004.

[37] F. Katzen, M. Deshmukh, F. Daldal, and J. Beckwith, "Evolutionary domain fusion expanded the substrate specificity of the transmembrane electron transporter DsbD," $E M B O$ Journal, vol. 21, no. 15, pp. 3960-3969, 2002.

[38] R. A. Kimball, L. Martin, and M. H. Saier Jr., "Reversing transmembrane electron flow: the DsbD and DsbB protein families," Journal of Molecular Microbiology and Biotechnology, vol. 5, no. 3, pp. 133-149, 2003.

[39] T. Frickey and A. N. Lupas, "Phylogenetic analysis of AAA proteins," Journal of Structural Biology, vol. 146, no. 1-2, pp. 2-10, 2004.

[40] L. M. Iyer, D. D. Leipe, E. V. Koonin, and L. Aravind, "Evolutionary history and higher order classification of AAA+ ATPases," Journal of Structural Biology, vol. 146, no. 1-2, pp. 11-31, 2004.

[41] A. N. Lupas and J. Martin, "AAA proteins," Current Opinion in Structural Biology, vol. 12, no. 6, pp. 746-753, 2002.
[42] J. E. Schulz and A. Matin, "Molecular and functional characterization of a carbon starvation gene of Escherichia coli," Journal of Molecular Biology, vol. 218, no. 1, pp. 129-140, 1991. 

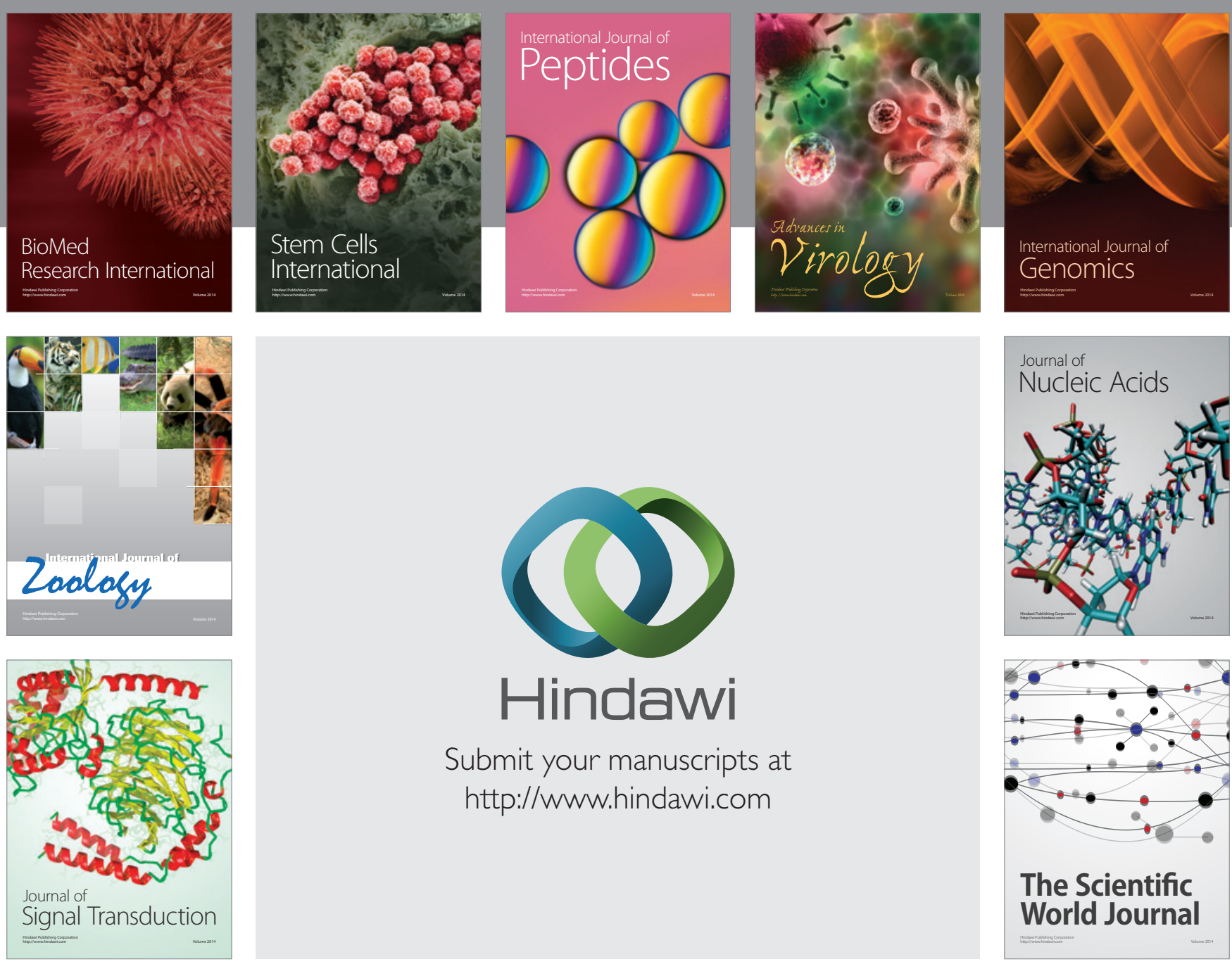

Submit your manuscripts at

http://www.hindawi.com
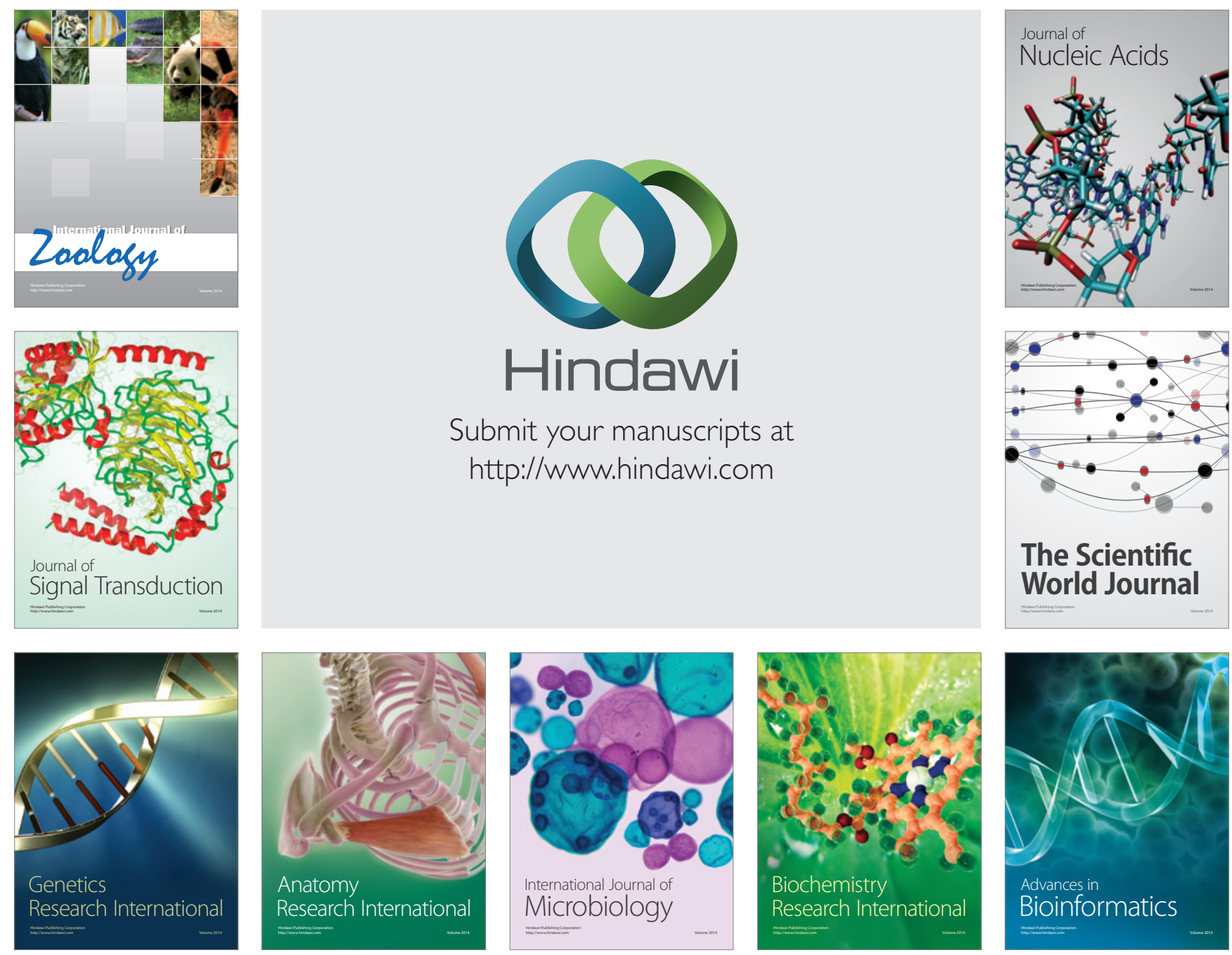

The Scientific World Journal
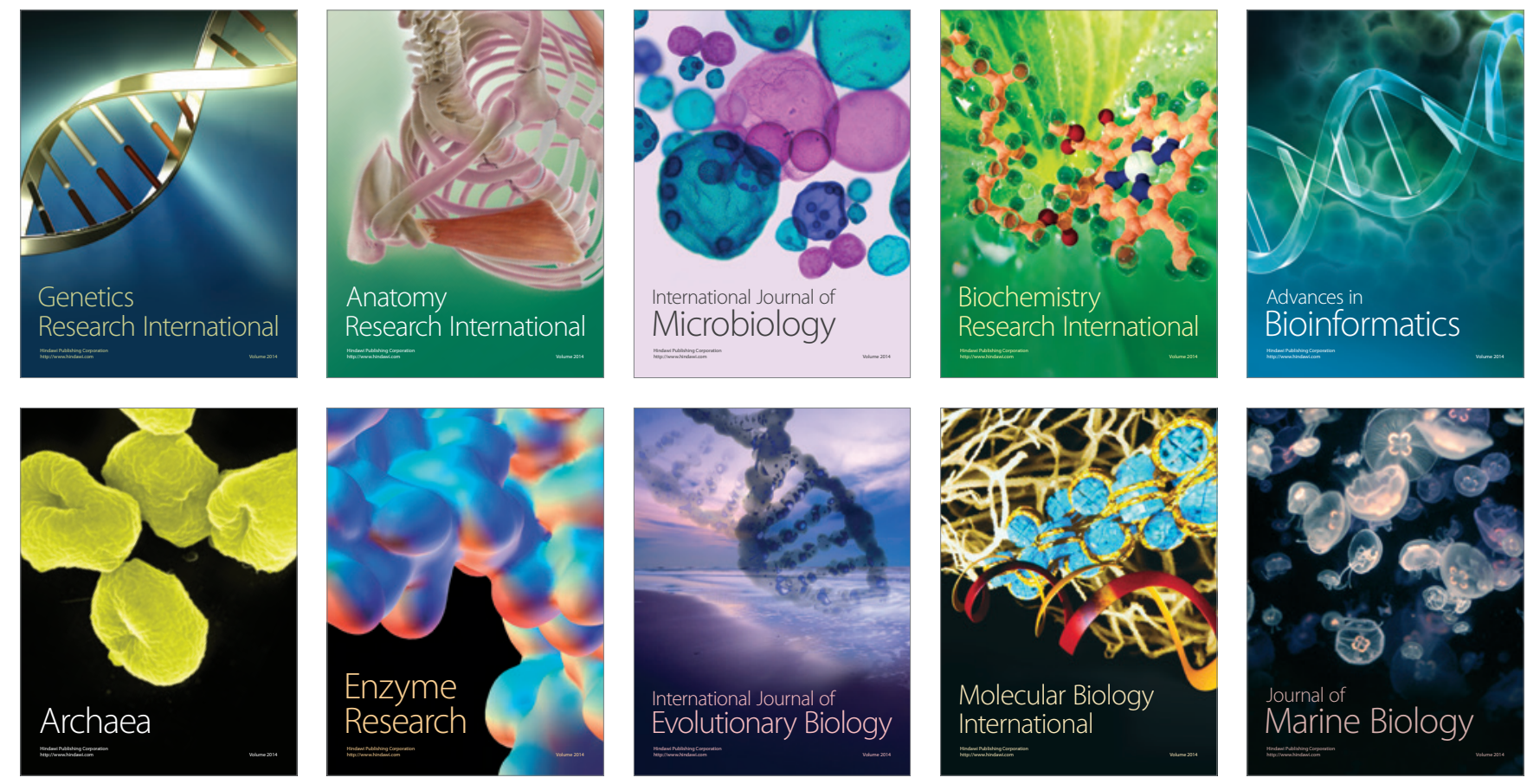